ARTICLE

httpst//doi.org/10.1038/s41467-020-16794-5

\title{
Autophagy controls the induction and developmental decline of NMDAR-LTD through endocytic recycling
}

Hongmei Shen (1) 1,2,3,4, Huiwen Zhu (i) ${ }^{1,4}$, Debabrata Panja (1) ${ }^{1,4}$, Qinhua Gu (1) ${ }^{1} \&$ Zheng Li (i) ${ }^{1 凶}$

NMDA receptor-dependent long-term depression (NMDAR-LTD) is a long-lasting form of synaptic plasticity. Its expression is mediated by the removal of AMPA receptors from postsynaptic membranes. Under basal conditions, endocytosed AMPA receptors are rapidly recycled back to the plasma membrane. In NMDAR-LTD, however, they are diverted to late endosomes for degradation. The mechanism for this switch is largely unclear. Additionally, the inducibility of NMDAR-LTD is greatly reduced in adulthood. The underlying mechanism and physiological significance of this phenomenon are elusive. Here, we report that autophagy inhibition is essential for the induction and developmental dampening of NMDAR-LTD. Autophagy is inhibited during NMDAR-LTD to decrease endocytic recycling. Autophagy inhibition is both necessary and sufficient for LTD induction. In adulthood, autophagy is upregulated to make LTD induction harder, thereby preventing the adverse effect of excessive LTD on memory consolidation. These findings reveal the unrecognized functions of autophagy in synaptic plasticity, endocytic recycling, and memory.

\footnotetext{
${ }^{1}$ Section on Synapse Development and Plasticity, National Institute of Mental Health, National Institutes of Health, Bethesda, MD 20892, USA. ${ }^{2}$ Present address: Key Laboratory of Neuroregeneration of Jiangsu and Ministry of Education \& Co-innovation Center of Neuroregeneration, Nantong University, Nantong 226001, China. ${ }^{3}$ Present address: Nantong Brain Hospital \& Mental Health Center Affiliated to Nantong University, Nantong University, Nantong 226005, China.

${ }^{4}$ These authors contributed equally: Hongmei Shen, Huiwen Zhu, Debabrata Panja. ${ }^{凶}$ email: lizheng2@mail.nih.gov
} 
S ynaptic plasticity, the ability of synapses to change in strength, is a cellular mechanism underlying information processing and storage in the brain. NMDA receptordependent long-term depression (NMDAR-LTD) is essential for contextual fear memory, spatial memory, contextual habituation, spatial reversal learning, behavioral flexibility, novelty acquisition, and social recognition ${ }^{1-9}$. Although NMDAR-LTD is can be induced by learning in adult animals, it is more readily induced in the first three postnatal weeks ${ }^{10-13}$. Little is known about how NMDAR-LTD is down-regulated in adults and the physiological significance of this phenomenon.

NMDAR-LTD is triggered by NMDA receptor activation which causes $\mathrm{Ca}^{2+}$ increase, activation of $\mathrm{Ca}^{2+}$-dependent protein phosphatases, and subsequent GluA1 dephosphorylation and activation of the mitochondria-caspase pathway ${ }^{14-16}$. These processes drive the removal of synaptic AMPA receptors. AMPA receptor removal begins with clathrin-dependent endocytosis, followed by sorting into either recycling endosomes (for reinsertion into the plasma membrane) or late endosomes (for degradation in lysosomes) ${ }^{17}$. While AMPA receptors rapidly recycle back to plasma membranes under basal conditions, they are diverted from recycling to late endosomes after NMDA receptor activation ${ }^{18,19}$. The mechanism for suppressing AMPA receptor recycling during NMDAR-LTD is largely unclear.

Macroautophagy (autophagy hereinafter) is a process through which proteins and organelles are delivered to lysosomes for degradation ${ }^{20,21}$. The best-studied function of autophagy is cellular homeostasis 22,23 . Neuronal autophagy also contributes to dopamine release, presynaptic assembly, axonal outgrowth, and dendritic spine pruning ${ }^{24-28}$. A role of autophagy in LTD has been hypothesized as NMDA receptor activation causes a delayed increase in autophagic flux in cultured hippocampal neurons ${ }^{29}$. This hypothesis, however, is incompatible with the finding that autophagy inhibitors have no effect on hippocampal LTD in mice until they are aged and accumulate Tau oligomers ${ }^{30}$. The role of autophagy in LTD remains elusive.

Here, we show that autophagic flux in CA1 neurons is transiently decreased during the induction phase of NMDAR-LTD. This autophagy inhibition causes a reduction of endocytic recycling and is required for AMPA receptor internalization and synaptic depression. Moreover, autophagy is upregulated during development to decrease the inducibility of NMDAR-LTD in adults to ensure proper consolidation of associative fear memory.

\section{Results}

Autophagy is inhibited in LTD. To test whether autophagy is involved in synaptic plasticity, we analyzed autophagy during LTP and LTD using two methods: time-lapse imaging and immunoblotting for LC3 and p62. The cleaved and phosphatidylethanolamine conjugated form of LC3 (LC3-II) is incorporated into autophagosomes and remains on mature autophagosomes ${ }^{31-33}$. LC3-II in combination with p62 (a protein selectively degraded by autophagy) indicates autophagic flux ${ }^{34-38}$.

For imaging, cultured hippocampal slices (7 days in vitro or DIV 7) were biolistically transfected to express monomeric red fluorescent protein-tagged LC3 (mRFP-LC3), mRFP-LC3G120A (lipidation-defective LC3 mutant that cannot be incorporated into autophagosomes, therefore exhibits a diffuse pattern of cellular expression), or GFP tagged p62 (GFP-p62). At 3-5 days after transfection, we stimulated the Schaffer collateral pathway (SC) with high-frequency stimulation (HFS; $100 \mathrm{~Hz}, 2$ trains of 100 pulses with an intertrain interval of $15 \mathrm{~s}$ ) for LTP induction or low-frequency stimulation (LFS; $1 \mathrm{~Hz}, 900$ pulses) for LTD induction. LFS decreased mRFP-LC3 puncta at all time points measured after stimulation and had no effect on mRFP-
LC3G120A (Fig. 1a-d). LFS also increased the integrated intensity of p62 at $15 \mathrm{~min}$ and $30 \mathrm{~min}$ after stimulation (Fig. 1e, f). HFS had no significant effect on mRFP-LC3 puncta, mRFPLC3G120A or p62 (Fig. 1a-f). These results indicate that LFS causes a transient inhibition of autophagy.

For immunoblotting, acute hippocampal slices from 16-19day-old mice were stimulated at SC with LFS or HFS. The CA1 region near the stimulating electrode was removed for immunoblotting. While LC3-II decreased and p62 increased at $15 \mathrm{~min}$ post-stimulation (Fig. 1g-i), they returned to pre-stimulation levels by $60 \mathrm{~min}$ post-stimulation (Fig. 1j-1). The hippocampal tissue used for immunoblotting contained neurites and somas, while only dendrites of pyramidal neurons were measured by imaging. This difference may account for the lack of LC3 changes in tissue lysates at $60 \mathrm{~min}$ poststimulation. The alteration of LC3II and p62 at $15 \mathrm{~min}$ and recovery by $60 \mathrm{~min}$ poststimulation was preserved in the presence of leupeptin (Fig. 1g-1), a nonlysosomotropic autophagy inhibitor, indicating that the autophagy inhibition is transient. Leupeptin increased both LC3-II and p62 in slices because of the inhibition of autophagic flux (Supplementary Fig. 1A-C). It is noted that because LC3-II is an intermediary protein in autophagy, autophagy impairment can cause either increase or decrease of LC3. HFS had no significant effect on LC3-II or p62 regardless of leupeptin treatment (Fig. 1g-1).

Taken together, these results show that autophagy is inhibited in LTD but not in LTP.

Autophagy inhibition is required for LTD induction. To examine the effect of autophagy inhibition on LTD, we activated autophagy in acute hippocampal slices with rapamycin which suppresses the initiation of autophagosome formation $19,39,40$. Rapamycin increased LC3-II and decreased p62 in hippocampal slices (Supplementary Fig. 1A-C). Field excitatory postsynaptic potentials (fEPSPs) were recorded in the CA1 region by stimulating SC. Rapamycin blocked LTD (fEPSPs at 50-60 min after LFS normalized to the pre-stimulation baseline: $70.54 \pm 3.61 \%$ in vehicle-treated and $95.71 \pm 3.62 \%$ in rapamycin-treated slices; two-tailed Student's $t$ test, $p=6.8 \times 10^{-20}$; Fig. $2 a$ ) and had no effect on LTP (fEPSPs at 50-60 min after HFS normalized to the pre-stimulation baseline: $133.33 \pm 3.59 \%$ in vehicle-treated and $136.46 \pm 4.23 \%$ in rapamycin-treated slices; two-tailed Student's $t$ test, $p=0.15$; Fig. $2 \mathrm{~b}$ ). The input-output relationship and pairedpulse ratio were intact in rapamycin-treated slices (Supplementary Fig. 1D, E).

To test whether rapamycin blocks LTD via autophagy and whether at pre- or post-synaptic sites, we generated CA1-specific and CA3-specific Atg5 knockout mice by crossing floxed Atg5 mice with mice expressing Cre selectively in the CA1 or CA3 region ${ }^{41,42}$. In P19 mice, the reduction of Atg5 in the CA1 area of Atg5flox/flox CA1Cre ${ }^{+}$mice and in the CA3 area of Atg 5 flox/flox $^{\mathrm{CA} 3 \mathrm{Cre}}{ }^{+}$mice were comparable (Supplementary Fig. 1F-H).

Rapamycin blocked LTD in CA1-Cre ${ }^{-}$and CA3-Cre ${ }^{+}$slices, but not in CA1-Cre ${ }^{+}$slices [fEPSPs at 50-60 min after LFS normalized to the pre-stimulation baseline: $95.60 \pm 0.46 \%$ in $\mathrm{Cre}^{-}$slices; $95.16 \pm 0.35 \%$ in $\mathrm{CA}_{3}-\mathrm{Cre}^{+}$slices; $80.52 \pm 0.49 \%$ in $\mathrm{CA}^{-}-\mathrm{Cre}^{+}$ slices; one-way ANOVA $\left(p=1.3 \times 10^{-20}\right)$ and post hoc Bonferroni $\left(p=1.7 \times 10^{-19}\right.$ for CA1-Cre ${ }^{+}$vs. $\mathrm{Cre}^{-} ; p=3.7 \times 10^{-19}$ for CA3$\mathrm{Cre}^{+}$vs. CA1-Cre ${ }^{+}$) were used for statistical analysis; Fig. 2c]. LTP, the input-output relationship, and paired-pulse ratio were intact in CA1-specific Atg5 knockout mice (Fig. 2d, Supplementary Fig. 1I, J). Since CA1 neurons are postsynaptic while CA3 neurons are presynaptic in SC synapses, these results indicate that rapamycin inhibits LTD through postsynaptic autophagy. 
a

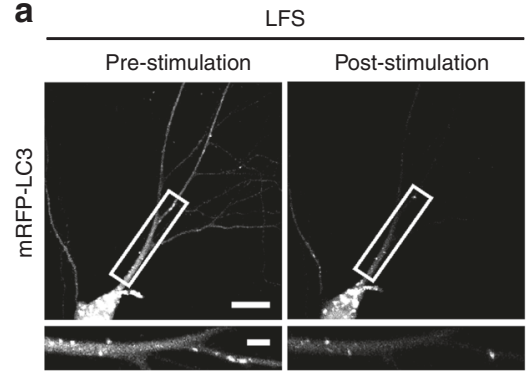

C

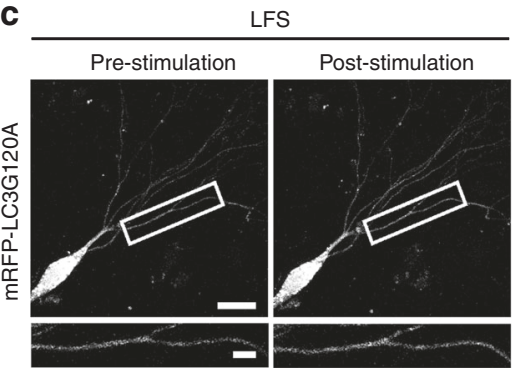

e

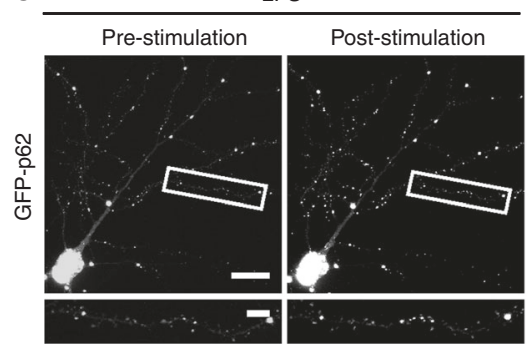

g

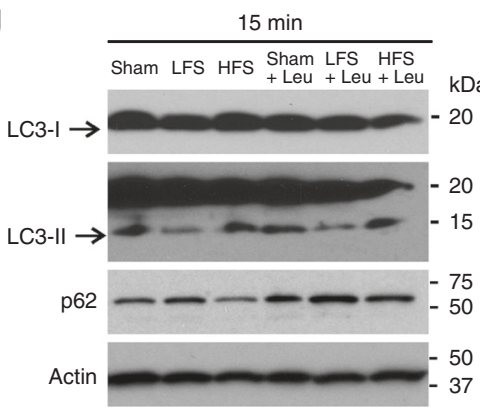

$\mathbf{j}$

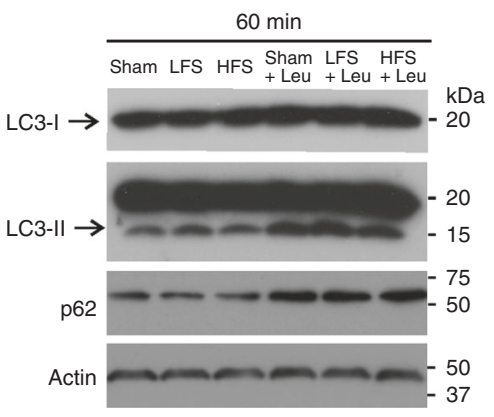

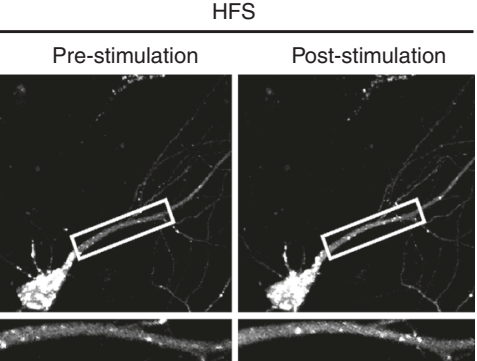
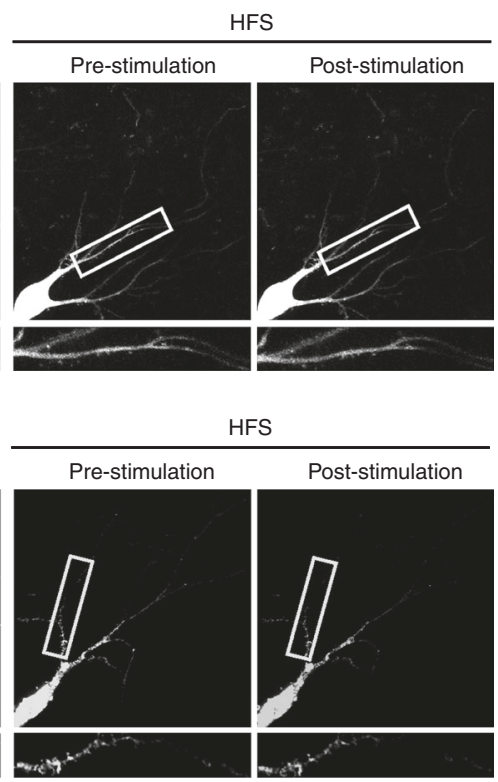

h
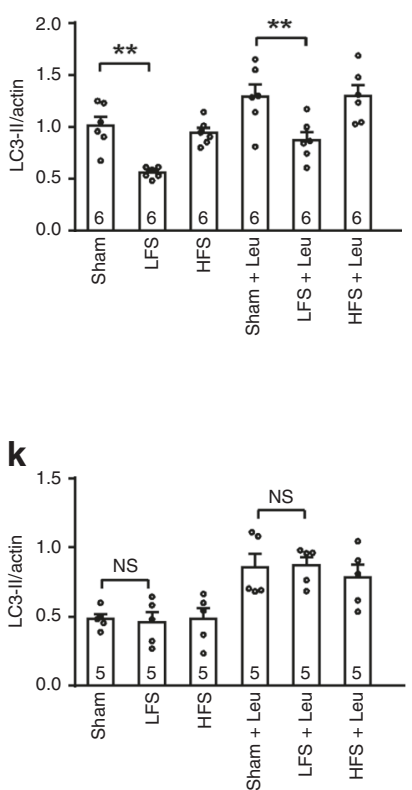

b

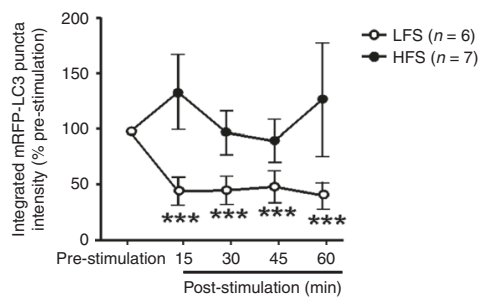

d
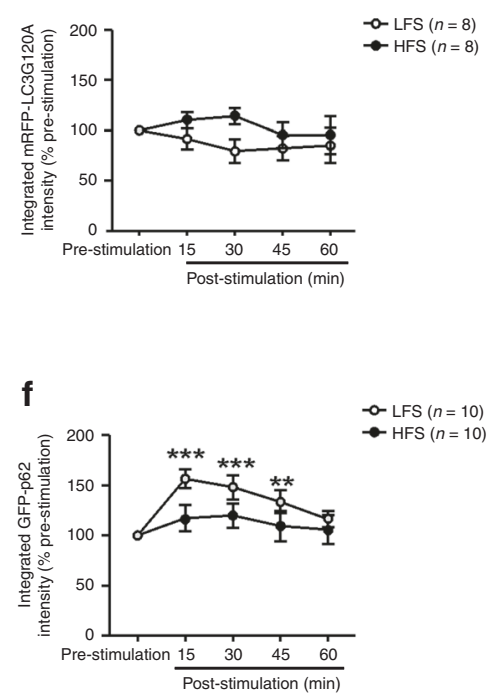

i
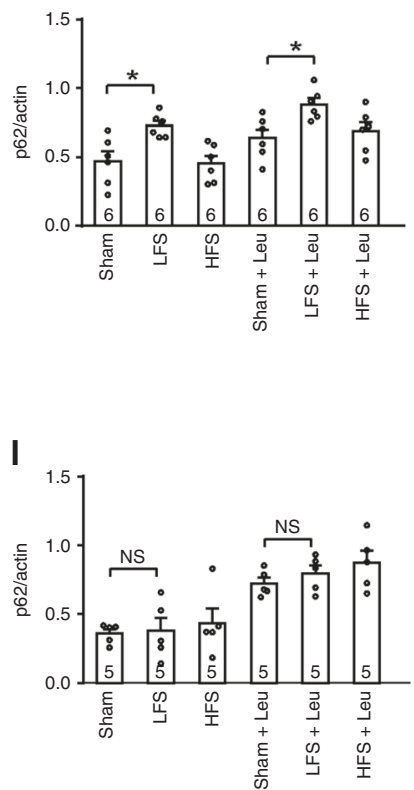

Moreover, we inhibited autophagy by using Beclin-1 siRNAs. The efficacy and specificity of Beclin-1 siRNAs were tested in primary hippocampal neurons (Supplementary Fig. $1 \mathrm{~K}-\mathrm{N}$ ). Lentivirus expressing Beclin-1 siRNAs, but not scrambled oligonucleotides, blocked the effect of rapamycin (fEPSPs at 50-60 min after LFS normalized to the pre-stimulation baseline:
$102.5 \pm 4.2 \%$ in scramble slices treated with rapamycin, $48.0 \pm$ $5.2 \%$ in scramble slices treated with vehicle, two-tailed Student's $t$ test, $p=0.000001 ; 48.5 \pm 4.4 \%$ in Beclin- 1 siRNA slices treated with rapamycin, $46.4 \pm 4.7 \%$ in Beclin- 1 siRNA slices treated with vehicle, two-tailed Student's $t$ test, $p=0.75$; Fig. 2e, f). Hence, rapamycin inhibits LTD via autophagy. 
Fig. 1 Autophagy is inhibited by low-frequency stimulation that induces LTD. a, c, e Representative images of the same dendrites of CA1 neurons in cultured hippocampal slices before and after stimulation at the Schaffer collateral pathway with low-frequency stimulation (LFS) or high-frequency stimulation (HFS); scale bars, $20 \mu \mathrm{m}$ in the upper images and $5 \mu \mathrm{m}$ in the lower images. b, d, f Quantification of integrated fluorescence intensity for mRFPLC3 puncta in a, mRFP-LC3G120A in c, and GFP-p62 in e; one-way RM ANOVA or Kruskal-Wallis one-way RM ANOVA on Ranks was used to compare different time points within the HFS or LFS group (b: $p=9.4 \times 10^{-5}$ for LFS, $p=0.704$ for HFS; d: $p=0.454$ for LFS, $p=0.570$ for HFS; f: $p=2.35 \times 10^{-7}$ for LFS, $p=0.399$ for HFS); Holm-Sidak was used to identify the post-stimulation time points significantly different from the pre-stimulation level in the LFS group (indicated by asterisks); $n$ indicates the number of slices, and one slice per animal was used. $\mathbf{g}$, $\mathbf{j}$ Representative blots of CA1 tissues taken from acute hippocampal slices stimulated with LFS or HFS at the Schaffer collateral pathway. h, i Quantification of LC3-II and p62 for $\mathbf{g}$. k, I Quantification of LC3-II and p62 for $\mathbf{j}$. In $\mathbf{g}-\mathbf{I}$, the number in the bars indicates the number of animals for each condition; one-way ANOVA was used to compare across groups (h: $p=5 \times 10^{-6} ; \mathbf{i}: p=0.00005 ; \mathbf{k}: p=0.000423 ; \mathbf{I}: p=5.30 \times 10^{-5}$; groups significantly different from the sham group were identified with Student-Newman-Keuls test and marked by asterisks. No adjustments were made for multiple comparisons Data are presented as mean $\pm \mathrm{SEM}$; ${ }^{\star} p<0.05$, ${ }^{\star \star} p<0.01,{ }^{\star \star \star} p<0.001$. Source data are provided as a Source Data file.

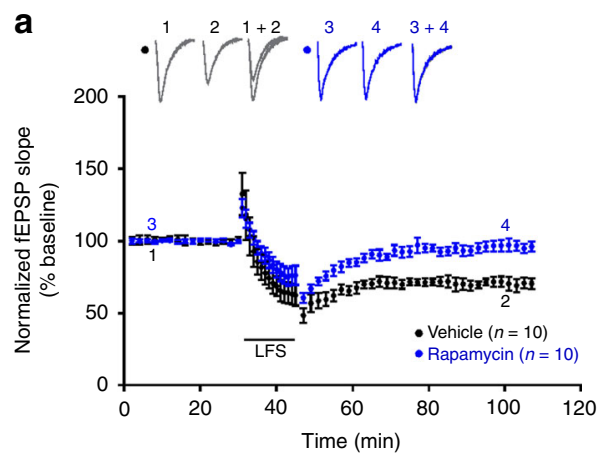

$\stackrel{\gtrless}{\bigsqcup_{20} \mathrm{~ms}}$
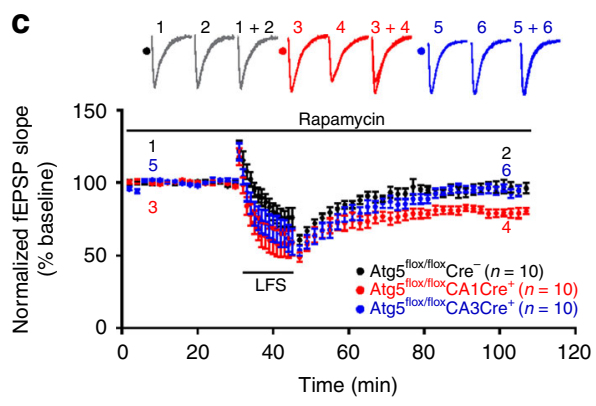

e
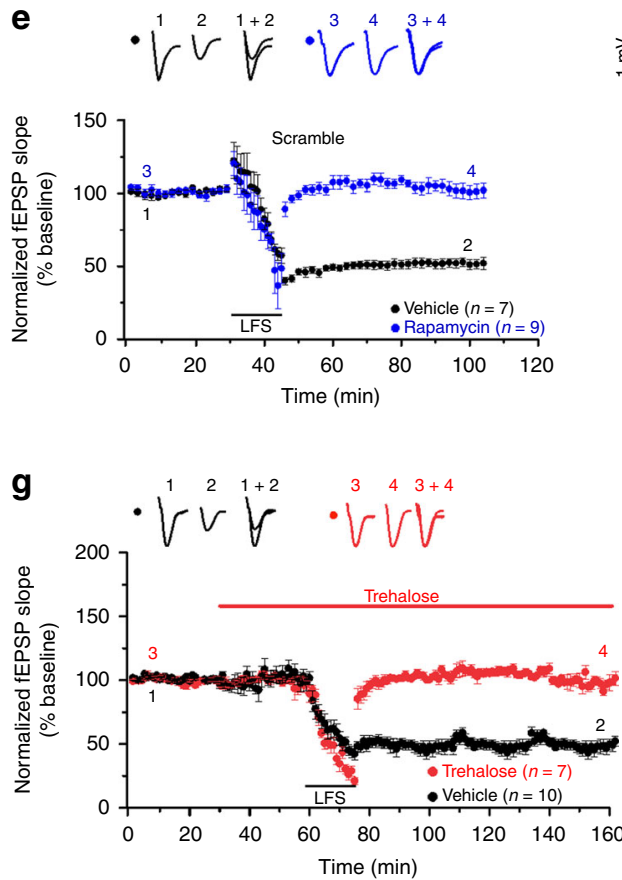

b
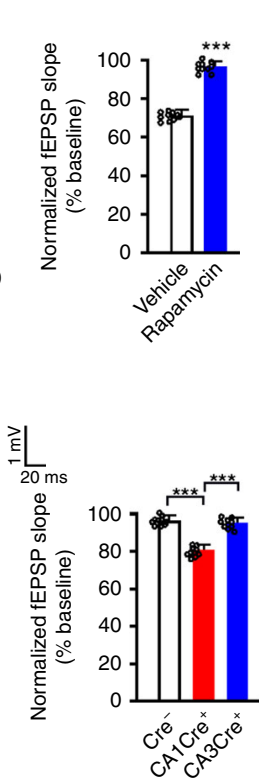

d

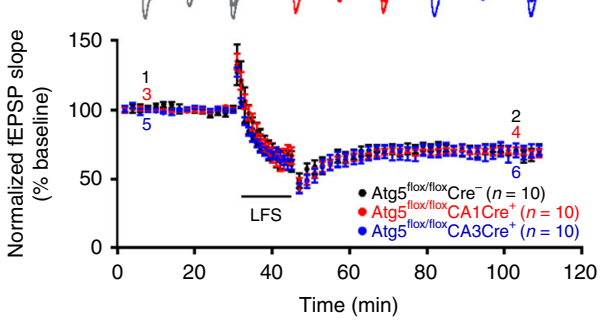

리니

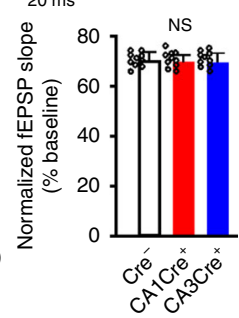

f
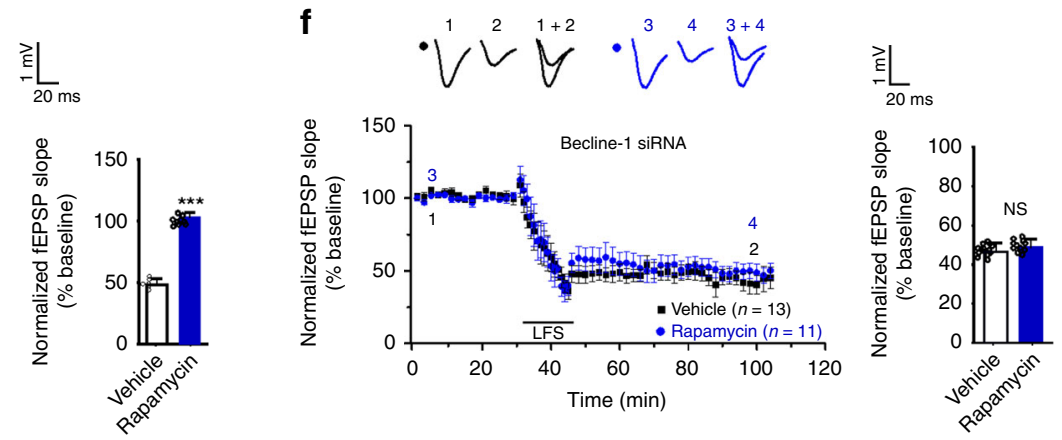

h

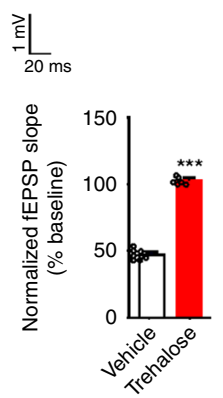

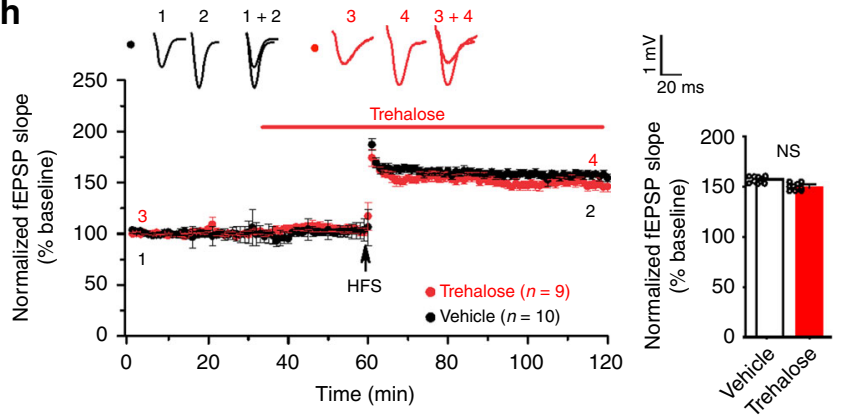


Fig. 2 Autophagy inhibition is required for LTD Induction. Acute hippocampal slices (a-d, $\mathbf{g}, \mathbf{h}$ ) or cultured hippocampal slices (e, $\mathbf{f}$ ) were stimulated at the Schaffer collateral pathway and recorded in the CA1 region for field excitatory postsynaptic potentials (fEPSPs). a, b Wild-type acute slices were perfused with vehicle or rapamycin $(1 \mu \mathrm{M})$ for 30 min before LFS (for LTD induction) or HFS (for LTP induction) and throughout the recording period; quantification on the right shows the average slope of fEPSPs recorded at 50-60 min after LFS or HFS normalized to the pre-stimulation baseline; twotailed Student's $t$ test was used for statistical analysis. c, d Acute slices taken from Atg $5^{f l o x} /{ }^{f l o x} \mathrm{Cre}^{+}$and Atg $5^{\text {flox/flox }} \mathrm{Cre}{ }^{-}$mice were stimulated with LFS; slices in c were perfused with rapamycin $(1 \mu \mathrm{M})$ for 30 min before LFS and throughout the recording period; quantification on the right shows the average slope of fEPSPs recorded at 50-60 min after LFS normalized to the pre-stimulation baseline; one-way ANOVA was used for comparison across groups; Bonferroni test was used to identify groups significantly different from the $A \operatorname{tg} 5^{\text {flox/flox }} \mathrm{Cre}^{-}$group in c; no adjustments were made for multiple comparisons. e, $\mathbf{f}$ Wild-type, cultured hippocampal slices were perfused with vehicle or rapamycin $(1 \mu \mathrm{M})$ for 30 min before LFS and throughout the recording period; quantification on the right shows the average slope of fEPSPs recorded at 50-60 min after LFS normalized to the pre-stimulation baseline; two-tailed Student's $t$ test was used for statistical analysis. $\mathbf{g}$, h Wild-type, acute slices were perfused with vehicle or trehalose (20 mM) for 30 min before LFS (for LTD induction in $\mathbf{g}$ ) or HFS (for LTP induction in $\mathbf{h}$ ) and throughout the recording period; quantification on the right shows the average slope of fEPSPs recorded at 50-60 min after LFS or HFS normalized to the pre-stimulation baseline; two-tailed Student's $t$ test was used for statistical analysis. Data are presented as mean $\pm \mathrm{SEM} ; n$ indicates the number of slices (one slice from each animal); ${ }^{\star \star \star} p<0.001$. LFS: low-frequency stimulation. HFS highfrequency stimulation. Source data are provided as a Source Data file.

To corroborate the necessity of autophagy inhibition in LTD, we treated slices with trehalose, an mTOR-independent autophagy inducer ${ }^{43-45}$. Consistent with the reports that trehalose activates autophagy and also blocks autophagic flux ${ }^{46}$, trehalose increased both LC3-II and p62 (Supplementary Fig. 1A-C). Trehalose blocked LTD but not LTP (fEPSPs at 70-80 min after LFS normalized to the pre-stimulation baseline: $102.9 \pm 2.0 \%$ in trehalose-treated slices, $47.2 \pm 0.7 \%$ in vehicle-treated slices, twotailed Student's $t$ test, $p=3.9 \times 10^{-15}$; fEPSPs at $50-60 \mathrm{~min}$ after HFS normalized to the pre-stimulation baseline: $148.8 \pm 3.5 \%$ in trehalose-treated slices, $152.7 \pm 0.9 \%$ in vehicle-treated slices, twotailed Student's $t$ test, $p=0.27$; Fig. $2 \mathrm{~g}, \mathrm{~h}$ ).

To test whether autophagy inhibition is sufficient for synaptic depression, we inhibited autophagy with chloroquine (CQ). CQ increased LC3-II and p62 in hippocampal slices, consistent with inhibition of autophagic flux (Supplementary Fig. 1A-C). Postsynaptic responses in CA1 neurons were reduced by CQ either added to the bath solution during field potential recording or infused -via the patch pipette during whole-cell recording [fEPSP was reduced by $60.55 \pm 3.81 \%$ at $50-60 \mathrm{~min}$ after adding CQ to the bath, two-tailed paired Student's $t$ test, $p=0.0001$ for before vs. after CQ treatment; EPSC was reduced by $34.65 \pm 0.65 \%$ at $30-35 \mathrm{~min}$ after breaking the cell membrane, two-tailed paired t-test, $p=0.0001$ for comparison with the baseline]. The effect of CQ was blocked by rapamycin in field recording [fEPSPs at 50-60 min after CQ treatment normalized to the pre-CQ baseline: $100.5 \pm 1.0 \%$ for rapamycin, $39.5 \pm 2.5 \%$ for CQ, $95.3 \pm 1.7 \%$ for CQ plus rapamycin; one-way ANOVA $\left(p=6.5 \times 10^{-20}\right)$ and Bonferroni for post hoc analysis $\left(p=4.3 \times 10^{-18}\right.$ for CQ plus rapamycin vs. CQ); Fig. 3a] and in whole-cell recording [EPSCs at 30-35 min after breaking in normalized to baseline: $39.5 \pm 2.5 \%$ for CQ, $100.3 \pm$ $7.3 \%$ for CQ plus rapamycin, $100.5 \pm 1.0 \%$ for rapamycin; one-way ANOVA $\left(p=5.5 \times 10^{-10}\right)$ and Bonferroni for post hoc analysis $\left(p=3.6 \times 10^{-9}\right.$ for CQ plus rapamycin vs. CQ; Fig. 3b]. Hence, autophagy inhibition in postsynaptic neurons is sufficient to induce synaptic depression.

Next, we applied CQ and LFS sequentially to test whether they induce synaptic depression via the same pathway. fEPSPs were reduced by CQ (fEPSP at 50-60 min after CQ treatment: $44.7 \pm$ $4.0 \%$; two-tailed Student's $t$ test was used for comparison with preCQ baseline, $p=0.0001$ ) and not further decreased by LFS (fEPSP at 50-60 min post LFS: $44.38 \pm 5.3 \%$; two-tailed Student's $t$ test was used for comparison with 50-60 min after CQ treatment, $p=0.96$; Fig. 3c). Likewise, CQ treatment following LFS did not further decrease fEPSP (fEPSP at 10-20 min post-LFS: $50.34 \pm 3.3 \%$; fEPSP at $80-90 \mathrm{~min}$ post-CQ: $55.57 \pm 4.5$; two-tailed Student's $t$ test, $p=0.35$; Fig. $3 \mathrm{c}$ ). These results indicate that CQ and LFS use a common pathway to induce synaptic depression.
To validate the effect of autophagy inhibition on synaptic responses, we treated slices with leupeptin and bafilomycin A1 (a vacuolar $\mathrm{H}^{+}$-ATPase inhibitor blocking the fusion between autophagosomes and lysosomes). Bafilomycin A1 inhibits autophagic flux in hippocampal slices (Supplementary Fig. 1A-C).

Like CQ, bafilomycin A1 and leupeptin reduced synaptic response (fEPSP after leupeptin treatment: $55.8 \pm 5.9 \%$, $p=0.000001$ vs. baseline; EPSC after breaking in to infuse leupeptin: $30.4 \pm 7.3 \%, p=9 \times 10^{-9}$ vs. baseline; fEPSP after bafilomycin A1 treatment: $52.4 \pm 4.6 \%, p=8.3 \times 10^{-7}$ vs. baseline; EPSC after breaking in to infuse bafilomycin A1: $21.4 \pm 5.4 \%$, $p=1.4 \times 10^{-10}$ vs. baseline; two-tailed Student's $t$ test; Fig. $3 \mathrm{~d}-\mathrm{g}$ ).

Taken together, these findings indicate that autophagy inhibition in postsynaptic CA1 neurons is both necessary and sufficient for LTD induction.

AMPAR internalization in LTD requires autophagy inhibition. LTD expression is mediated by the removal of AMPA receptors from synapses $14,17,47,48$. To test whether autophagy is involved in AMPA receptor internalization, cultured hippocampal neurons (DIV 17) were stimulated with NMDA $(30 \mu \mathrm{M}, 5 \mathrm{~min})$ to induce chemical LTD which shares molecular mechanisms with LFSinduced $\mathrm{LTD}^{49}$. The internalization of AMPA receptor subunit GluA2 was analyzed by using an antibody-based internalization assay ${ }^{15,16}$. NMDA-induced GluA2 internalization and this was blocked by rapamycin (Fig. 4a, c). Rapamycin treatment alone had no effect on GluA2 internalization and activated autophagy (Fig. 4a, c; Supplementary Fig. 2A-C).

To examine whether rapamycin blocks GluA2 internalization via autophagy, we transfected neurons with plasmids expressing Atg5 siRNAs. Atg5 siRNAs (but not a scrambled sequence) knocked down overexpressed Atg5 (but not Beclin-1) and this effect was abolished by co-expressing Atg5 resistant to Atg5 siRNAs (Atg5mut, carrying synonymous mutations in the siRNA binding site; Supplementary Fig. 2D, E). The Atg5 siRNA also effectively knocked down endogenous Atg5 (Supplementary Fig. 2F, G).

Atg5 siRNAs blocked the effect of rapamycin on NMDAinduced GluA2 internalization, and the blockade was obliterated by Atg5mut (Fig. 4b, d). Basal GluA2 internalization was unchanged by Atg5 siRNAs (Supplementary Fig. 2H, I). Scrambled siRNAs had no effect on NMDA-induced or basal GluA2 internalization (Fig. 4b, d; Supplementary Fig. 2H, I). Hence, rapamycin blocks NMDA-induced GluA2 internalization through autophagy. Moreover, trehalose which increased both LC3-II and p62 as in hippocampal slices blocked NMDA-induced but not basal GluA2 internalization (Supplementary Fig. 2A-C, 
a
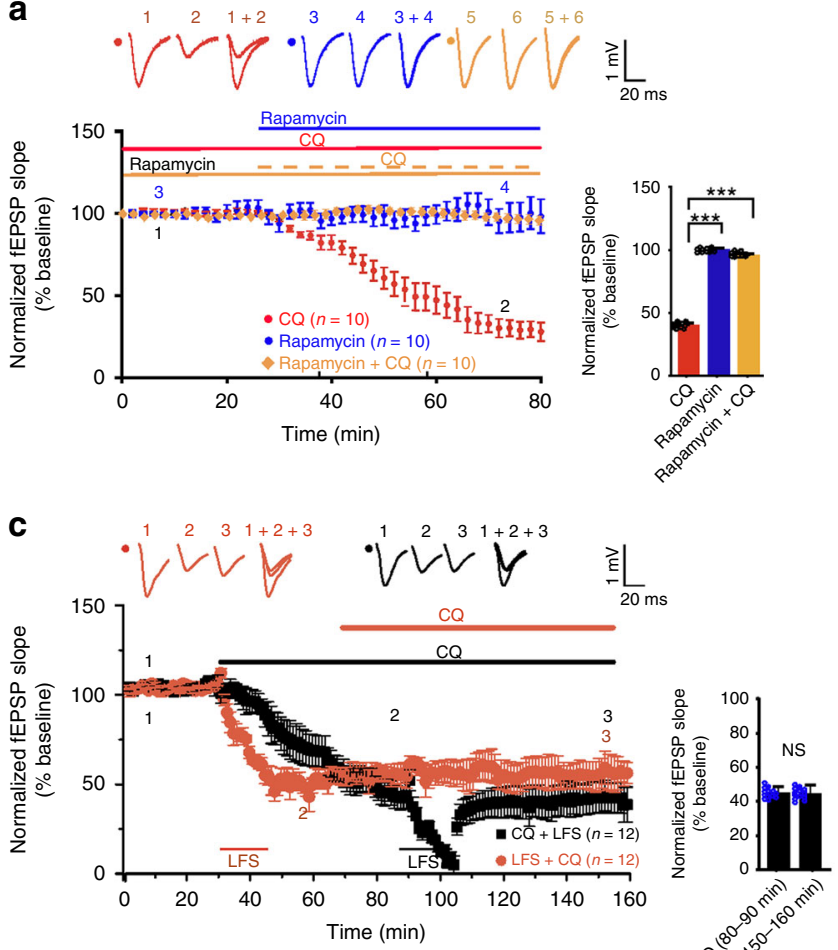

d
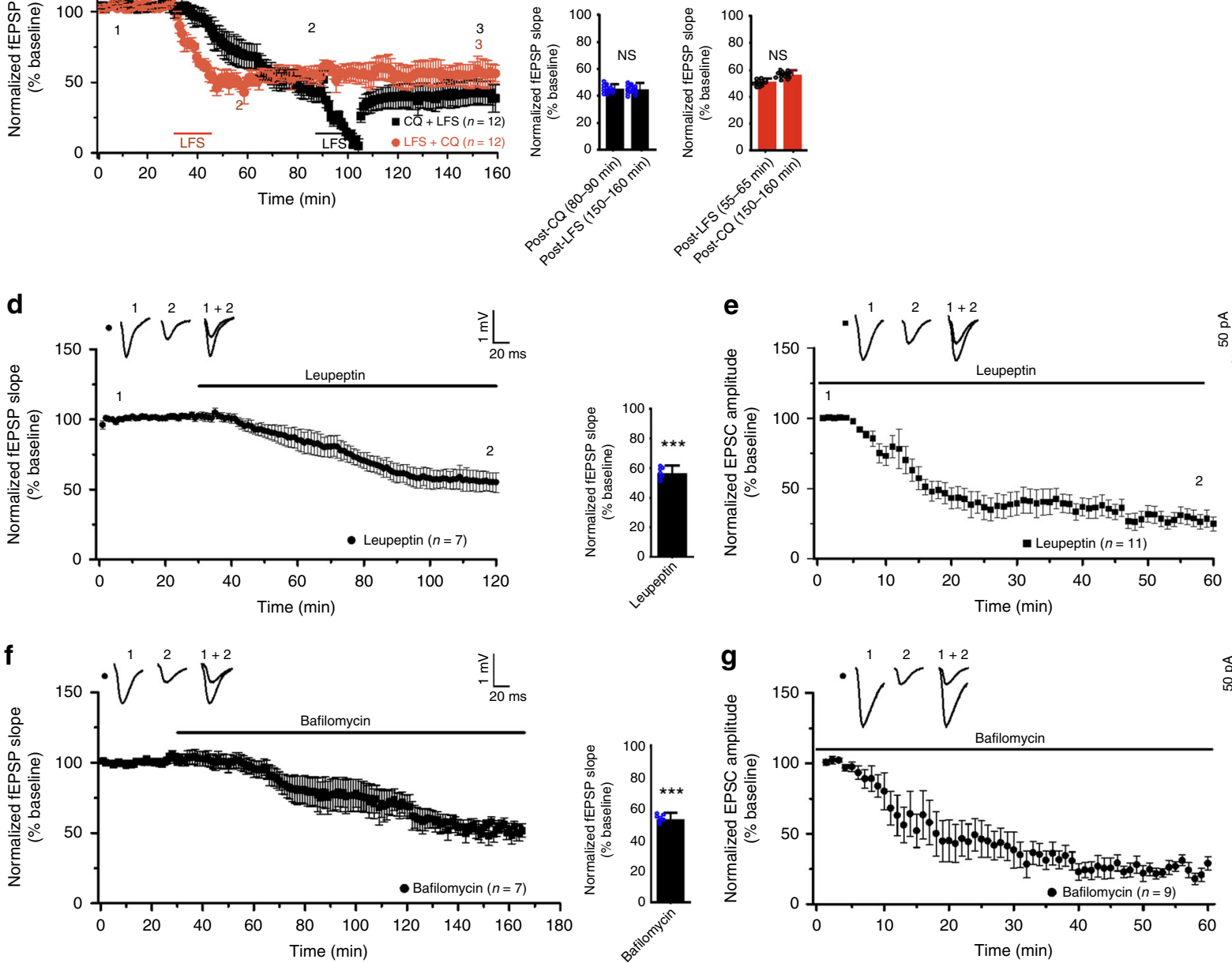

竞

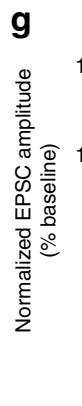

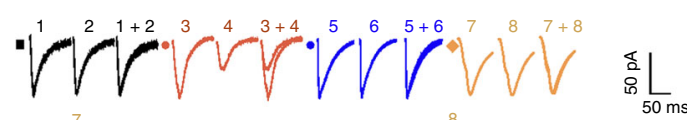

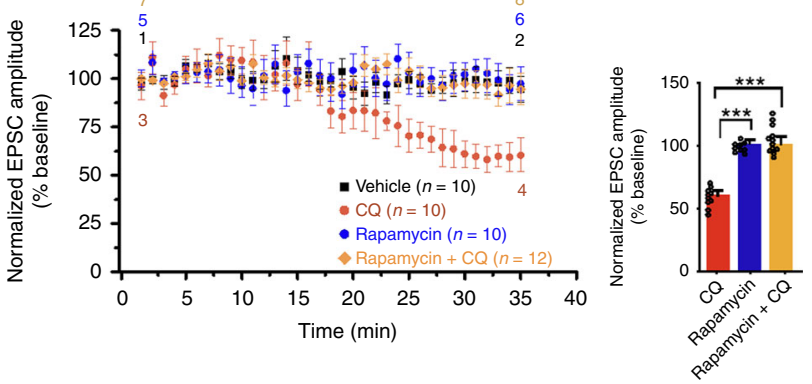

Fig. $4 \mathrm{a}, \mathrm{c}$ ). These results indicate that autophagy inhibition is required for AMPA receptor internalization during LTD.

To test whether inhibiting autophagy is sufficient to induce AMPA receptor internalization, we treated hippocampal neurons with CQ. CQ increased both LC3-II and p62 in cultured neurons (Supplementary Fig. 2A-C). It increased GluA2 internalization and occluded the effect of NMDA on GluA2 internalization (Supplementary Fig. 3). Hence, CQ and NMDA induce GluA2 internalization via the same pathway. Moreover, we inhibited autophagy with bafilomycin A1 and leupeptin. Both increased LC3-II, p62, and GluA2 internalization in neurons (Supplementary Fig. 2A-C;
Supplementary Fig. 3). These results indicate that autophagy inhibition is sufficient to induce GluA2 internalization.

Taken together, these findings indicate that autophagy inhibition is both necessary and sufficient for AMPA receptor internalization in LTD.

Autophagy regulates endocytic recycling in LTD. Since AMPA receptors are transported in endosomes during LTD, we proceeded to test whether autophagy affects endosomal trafficking. We transfected hippocampal neurons (DIV 14) with plasmids 
Fig. 3 Autophagy inhibitors induce synaptic depression. a Field excitatory postsynaptic potentials (fEPSPs) evoked by stimulation at the Schaffer collateral pathway were recorded in the CA1 region of acute hippocampal slices; CQ $(120 \mu \mathrm{M})$, rapamycin $(1 \mu \mathrm{M})$, or CQ plus rapamycin was added to the bath; quantification on the right shows the average slope of fEPSPs recorded at 50-60 min after drug perfusion normalized to the pre-treatment baseline for the CQ and the rapamycin group, or to the pre-CQ baseline for the CQ plus rapamycin group; one-way ANOVA was used for statistical analysis and Bonferroni was used for post hoc analysis. b Excitatory postsynaptic currents (EPSCs) recorded in CA1 neurons in whole-cell mode; CQ (120 $\mu$ M), rapamycin $(1 \mu \mathrm{M})$, or CQ plus rapamycin was infused through the patching pipette; quantification on the right shows the average amplitude of EPSCs at 30-35 min normalized to that recorded at 0-5 min after breaking in; one-way ANOVA was used for comparison across groups; Bonferroni test was used for post hoc analysis. c CQ $(120 \mu \mathrm{M})$ and LFS were applied sequentially to slices; quantification on the right shows the average slope of fEPSPs (recorded in designated time frames after the beginning of recording) normalized to the pre-treatment baseline; two-tailed paired t-test was used for statistical analysis. Slices were perfused with leupeptin $(300 \mu \mathrm{M}$; d) or bafilomycin $\mathrm{A} 1(20 \mu \mathrm{M} ; \mathbf{f})$; quantification on the right shows the average slope of fEPSPs recorded at 80-90 min after drug perfusion normalized to the pre-treatment baseline; two-tailed Student's $t$ test was used for statistical analysis. CA1 neurons were infused with leupeptin $(300 \mu \mathrm{M} ; \mathbf{e})$ or bafilomycin $\mathrm{A} 1(20 \mu \mathrm{M} ; \mathbf{g})$; quantification on the right shows the average amplitude of EPSCs at $35-40$ min normalized to that recorded at $0-5 \mathrm{~min}$ after breaking in; two-tailed Student's $t$ test was used for statistical analysis. Data are presented as mean \pm SEM; $n$ indicates the number of slices (one slice from each animal); ${ }^{\star \star \star} p<0.001$; no adjustments were made for multiple comparisons. Source data are provided as a Source Data file.

expressing EGFP-tagged Rab proteins to label endosomes (Rab5 for early endosomes, Rab11 for recycling endosomes, Rab9 for late endosomes) along with mRFP-LC3. Neurons were imaged 3-5 days later before and after chemical LTD induction. NMDA decreased mRFP-LC3 puncta and Rab11-labeled recycling endosomes, increased Rab5-labeled early endosomes, and had no significant effect on Rab9-labeled late endosomes (Fig. 5a-d; Supplementary Fig. 4A, B).

To test whether autophagy inhibition contributes to the endosomal changes, we co-treated neurons with NMDA and rapamycin. While rapamycin had no effect on basal Rab11labeled recycling endosomes (Supplementary Fig. 4C, D), it blocked their changes and mRFP-LC3 puncta decrease induced by NMDA (Fig. 5a-c). Rapamycin had no significant effect on Rab5-labeled early endosomes in untreated or NMDA-stimulated cells (Supplementary Fig. 4C, D; Fig. 5a, d). Trehalose and NMDA co-treatment had the same effect on endosomes as rapamycin and NMDA (Supplementary Fig. 4E, F). These results indicate that autophagy inhibition in LTD is required to decrease endocytic recycling.

To consolidate this finding, we examined endosomes using live imaging in cultured hippocampal slices. In slices transfected with a construct expressing GluA2 tagged with Super Ecliptic pHluorin (SEP, displaying fluorescence only when it is on the cell surface $)^{50,51}$, LFS decreased GluA2 fluorescence, suggestive of GluA2 internalization (Fig. 5e, f). This decrease was blocked by rapamycin (Fig. 5e, f). LFS decreased Rab11 labeled recycling endosomes, increased Rab5 labeled early endosomes, and had no significant effect on Rab9 labeled late endosomes (Fig. 5e, g; Supplementary Fig. 4G-J). Rapamycin blocked LFS-induced alteration of Rab11 but not that of Rab5 (Fig. 5e, g; Supplementary Fig. 4G, H). In Atg5 knockout slices, rapamycin had no effect on LFS-induced GluA2 internalization and the recycling endosome decrease (Fig. 5e-g), indicating that LFS induces AMPA receptor internalization and reduces recycling endosomes in an autophagy-dependent manner. Moreover, CQ and leupeptin decreased Rab11-labeled recycling endosomes (Fig. 5h, i), indicating that autophagy inhibition is sufficient to decrease recycling endosomes. To test whether the reduction of recycling endosomes contributes to autophagy inhibition, we used constitutively active Rab11 (CA-Rab11) to abate the recycling endosome decrease. CA-Rab11 increased transferrin uptake (Supplementary Fig. 5A, B), confirming that it promotes endocytic recycling. Despite more recycling endosomes, mRFP-LC3 puncta still decreased (Fig. 5j, k). Hence, autophagy inhibition in LTD is not caused by the reduction of recycling endosomes.

Fewer recycling endosomes in NMDA-treated cells suggest reduced endosomal recycling. This is confirmed by the transferrin assay (Supplementary Fig. 5C, D). NMDA-induced decrease in transferrin uptake was blocked by rapamycin, and the rapamycin effect was obliterated by Atg5 siRNAs (Supplementary Fig. 5C, D). Hence, endosomal recycling is reduced by NMDA treatment through autophagy inhibition.

To test if autophagy affects the endocytic trafficking of GluA2, we assessed the colocalization of GluA2 with EEA1 (an early endosome marker), LAMP1 (a late endosome marker), or transferrin. Hippocampal neurons (DIV 17) were incubated with the GluA2 antibody alone or along with transferrin. After rinse to remove unbound antibodies and transferrin, neurons were left $15 \mathrm{~min}$ to allow for transferrin and antibody-labeled surface GluA2 to be internalized. GluA2 was detected in EEA1, transferrin, and lamp1 positive structures (Fig. 6). Rapamycin and trehalose had no significant effect on the distribution of GluA2 in endosomes (Fig. 6). CQ and leupeptin decreased GluA2 in recycling endosomes, increased GluA2 in late endosomes, and had no effect on GluA2 in early endosomes (Fig. 6).

We also examined GluA2 distribution in Atg $5^{\text {flox/flox }}$ neurons transduced with AAV Cre which had decreased Atg5 expression (Supplementary Fig. 6A, B). These neurons had less GluA2 in recycling endosomes, more GluA2 in late endosomes, and no change to GluA2 in early endosomes (Supplementary Fig. 6C-F). These results indicate that autophagy inhibition reduces GluA2 in recycling endosomes.

To test if Rab11-containing recycling endosomes are involved in the endocytic trafficking of GluA2, we transfected cultured neurons (DIV 14) with the EGFP-Rab11 construct and analyzed GluA2 in endocytic trafficking 3 days later. A fraction of internalized GluA2 colocalized with Rab11, and the colocalization was increased by NMDA (Supplementary Fig. 6G, H). Hence, GluA2 is transported to Rab11-containing recycling endosomes during LTD.

Why endocytic recycling is reduced when autophagy is inhibited? Atg16L and mAtg9A-containing endocytic vesicles resulting from endocytosis from plasma membranes meet in recycling endosomes to form autophagosome precursors ${ }^{52,53}$. Since Atg16L and $\mathrm{mAtg} 9$ in recycling endosomes are en route to autophagosomes, inhibition of autophagic flux may cause accumulation of Atg16L and mAtg9 in recycling endosomes, thereby reducing endocytic recycling. Indeed, the proportion of Atg16L and mAtg9 in Rab11 positive structures increased in cells treated with CQ, leupeptin or bafilomycin A1, and it was unchanged by rapamycin or co-treatment with rapamycin and CQ (Fig. 7). NMDA also increased Atg16L and mAtg9 in Rab11 labeled recycling endosomes (Fig. 7).

Taken together, these findings indicate that autophagy inhibition during LTD leads to a suppression of endosomal recycling, which presumably facilitates the removal of AMPA receptors from plasma membranes. 
a

Sham

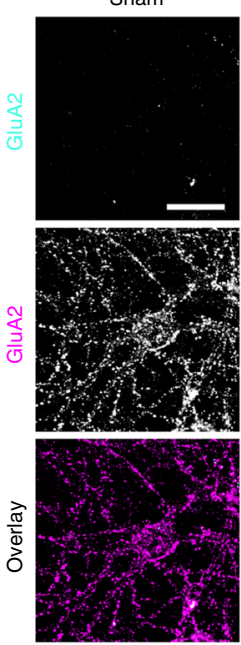

b
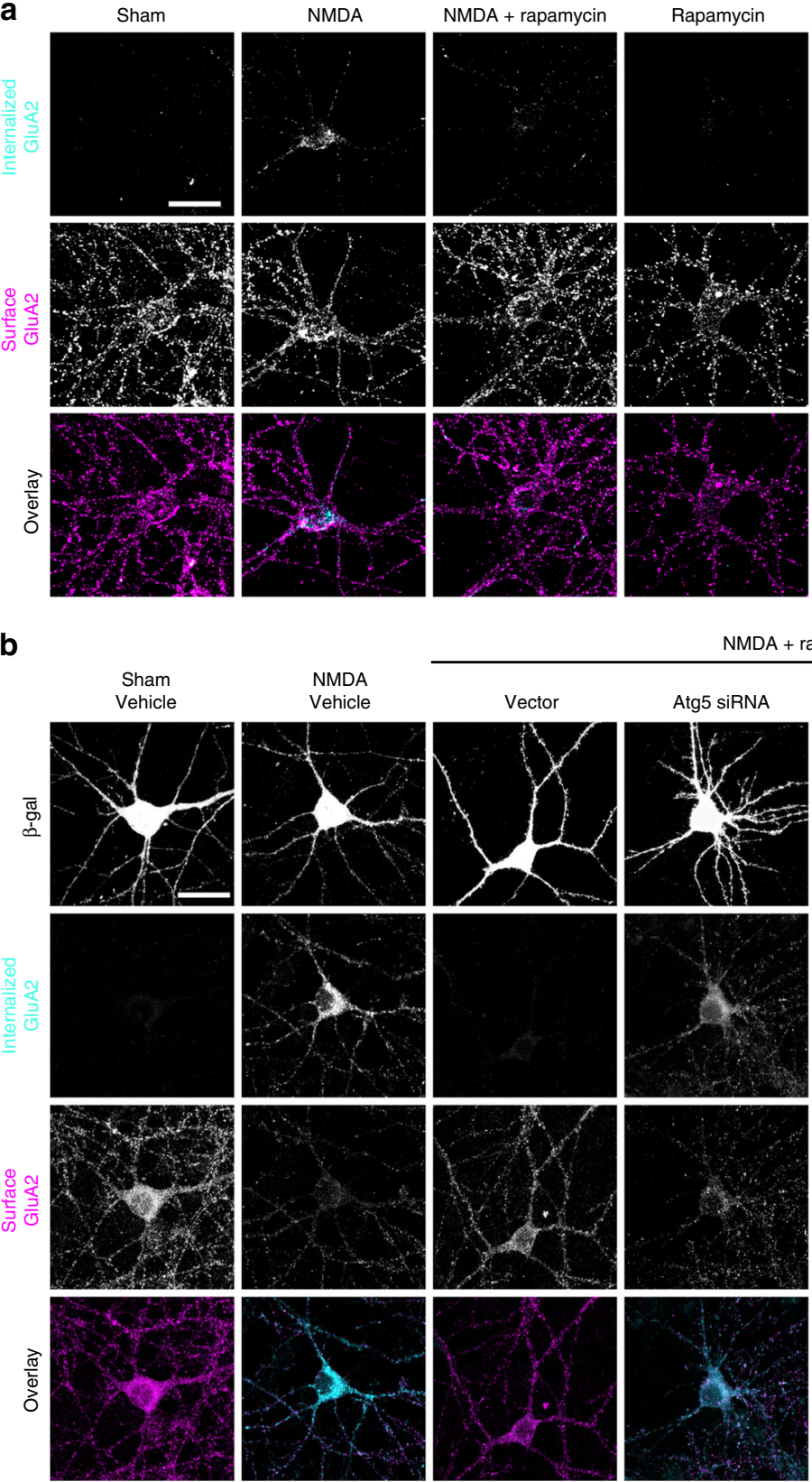

NMDA + trehalose
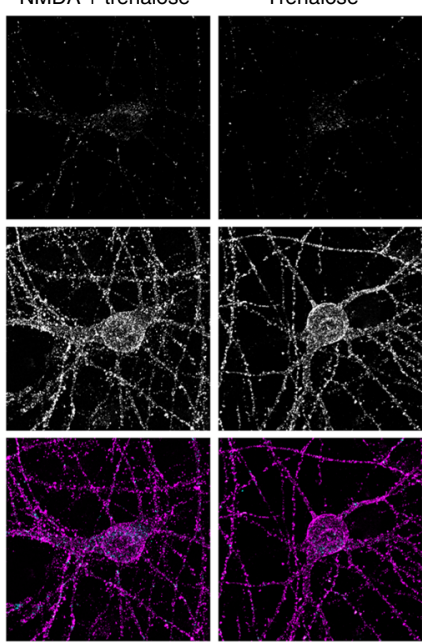

NMDA + rapamycin

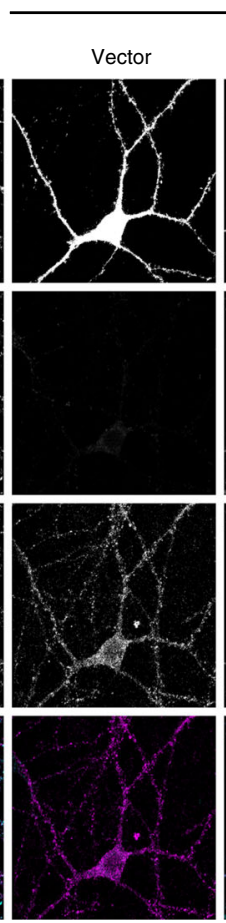

Atg5 siRNA

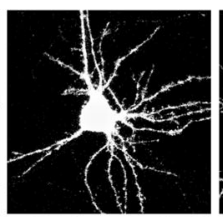

Scramble

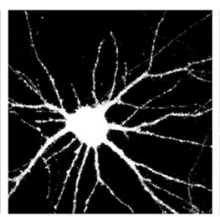

Atg5 siRNA
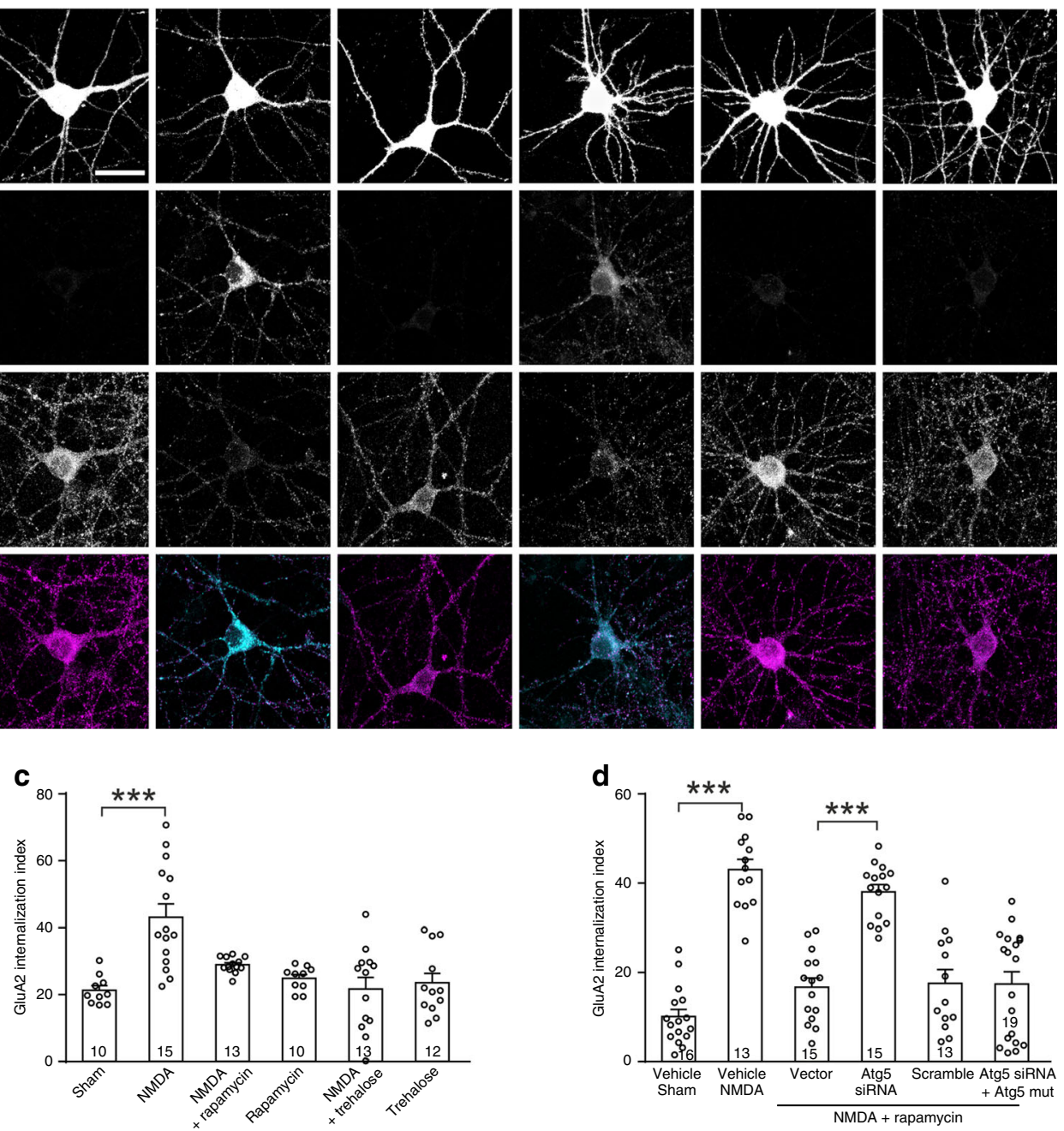

Autophagy is inhibited by active caspase-3 in LTD. NMDA receptor-dependent LTD and AMPA receptor internalization in hippocampal neurons require caspase- 3 activation ${ }^{16}$. To examine the relationship between autophagy and caspase- 3 in LTD, we treated cultured neurons with NMDA along with rapamycin or the caspase- 3 inhibitor DEVD-fmk. The effects of NMDA on LC3-II and p62 were blocked by DEVD-fmk (Fig. 8a-d). Rapamycin, by contrast, had no effect on NMDA-induced increase in cleaved, active caspase-3 (Fig. 8e, f). Both caspase-3 activation and autophagy inhibition induced by NMDA were abolished by the 
Fig. 4 Autophagy inhibition is required for AMPA receptor internalization during LTD. Untransfected primary hippocampal neurons (a, c) and neurons transfected with designated constructs $(\mathbf{b}, \mathbf{d})$ were treated with NMDA (30 $\mu \mathrm{M}, 5 \mathrm{~min})$, rapamycin $(1 \mu \mathrm{M}, 10 \mathrm{~min})$ and trehalose (20 mM, $30 \mathrm{~min})$ alone or in combination as indicated. a, b Representative images; scale bar, $20 \mu \mathrm{m}$. c Quantification of GluA2 internalization index [(integrated fluorescence intensity of internalized GluA2)/(total integrated fluorescence intensity of internalized and surface GluA2)] in a; Kruskal-Wallis one-way ANOVA on ranks was used to compare across groups $(p=0.000062)$; groups significantly different from the sham group were identified with Dunn's test and marked with asterisks. d Quantification of GluA2 internalization in b; Kruskal-Wallis one-way ANOVA on ranks was used to compare across groups $\left(p=8.5402 \times 10^{-11}\right)$; Dunn's test was used to identify significantly different groups; no adjustments were made for multiple comparisons. The graph shows mean \pm SEM; the number in the bar indicates the number of cells; ${ }^{\star \star \star} p<0.001$. Source data are provided as a Source Data file.

NMDA receptor antagonist (2R)-amino-5-phosphonopentanoate (AP5; Fig. 8a-f). These results indicate that autophagy inhibition in LTD requires caspase- 3 activity.

Several Atg proteins are caspase- 3 substrates ${ }^{54}$. In hippocampal slices treated with NMDA, the reported caspase- 3 substrates Atg3, 4B, 5, and 7 decreased, and the decrease was blocked by DEVD-fmk (Fig. 8g, h), suggesting that caspase-3 activation in LTD causes proteolysis of Atg proteins. To confirm that caspase-3 indeed inhibits autophagy, we measured LC3-II and p62 in caspase-3 knockout mice. LC3-II increased, while p62 decreased in the CA1 region of caspase- 3 knockout mice (Fig. 8i-k). Also, Atg3, 4B, 5, and 7 increased in caspase- 3 knockout mice (Fig. 8l, $\mathrm{m})$. In the CA1 region of $\mathrm{Atg} 5^{\text {flox/flox }} \mathrm{CA} 1 \mathrm{Cre}^{+}$mice and the CA3 region of $\mathrm{Atg} 5^{\text {flox/flox }} \mathrm{CA} 3 \mathrm{Cre}^{+}$mice, full-length and cleaved caspase- 3 were unchanged (Fig. $8 n-p$ ). These results suggest that caspase-3 inhibits autophagy, at least in part, by cleaving Atg proteins.

To test whether autophagy mediates the function of caspase- 3 in LTD, we infused active caspase- 3 alone or along with rapamycin via the whole-cell patch pipette into CA1 neurons. Active caspase- 3 decreased EPSCs and this effect was obliterated by co-infusion with rapamycin [average EPSCs at 30-35 min normalized to that at $0-5 \mathrm{~min}$ after break-in: $64.59 \pm 1.44 \%$ for active caspase- 3 and $98.42 \pm 1.56 \%$ for active caspase- 3 plus rapamycin, one-way ANOVA $\left(p=3.6 \times 10^{-18}\right)$ and post hoc Bonferroni ( $p=1 \times 10^{-16}$ for active caspase- 3 vs. active caspase- 3 plus rapamycin); Fig. 8q, r]. Heated, deactivated caspase- 3 or rapamycin alone had no effect on EPSCs [98.82 $\pm 1.10 \%$ for deactivated caspase- 3 and $99.99 \pm 0.59 \%$ for rapamycin, post hoc Bonferroni ( $p=7.4 \times 10^{-17}$ for deactivated caspase- 3 vs. active caspase-3); Fig. 8q, r; Fig. 3b]. Hence, autophagy inhibition is required for caspase-3-induced synaptic depression.

To test if caspase-3 activity is required for the recycling endosome decrease in LTD, we transfected cultured hippocampal neurons with the Rab11 construct and induced chemical LTD in the presence of DEVD-fmk. Rab11-labeled recycling endosomes were unchanged by NMDA (Supplementary Fig. 7), indicating that caspase- 3 activity is required to reduce endocytic recycling in LTD.

Taken together, these findings indicate that autophagy is inhibited by caspase- 3 and mediates the function of caspase- 3 in LTD.

Autophagy increases in adults to reduce LTD inducibility. The inducibility of NMDAR-LTD in SC synapses is much lower in adults than in juveniles ${ }^{10,12,13}$. To test if autophagy is involved in this phenomenon, we first examined the developmental pattern of autophagy in the CA1 region. LC3-II increased, while p62 decreased in adulthood (Fig. 9a-d). This developmental change was also present in hippocampal slices treated with leupeptin (Fig. 9e-h). By contrast, both full-length and active caspase-3 decreased in adulthood (Fig. 9i-k). To test whether the caspase decrease in adult hippocampi contributes to the autophagy increase, we treated adult hippocampal slices (10-week-old) with the cell-permeable caspase-3 activator AA2. AA2 decreased LC3-
II and increased p62 (Fig. 9l-n). Hence, the autophagy increase in adults is attributable, at least in part, to diminished caspase-3.

We next tested LTD in adult CA1 and CA3-specific Atg5 knockout mice ( 8 weeks of age). While LFS did not induce noticeable LTD in wild-type or CA3-specific Atg5 knockout slices, it induced LTD in CA1-specific Atg5 knockout slices (fEPSPs at 50-60 min post-LFS normalized to the pre-stimulation baseline: $102.19 \pm 0.85 \%$ in wild-type slices, $96.1 \pm 1.17 \%$ in CA3specific Atg5 knockout slices, $76.82 \pm 0.50 \%$ in CA1-specific Atg 5 knockout slices; one-way ANOVA, $p=3.8 \times 10^{-28}$; Fig. 10a). HFS-induced LTP was unchanged in CA1 or CA3-specific Atg5 knockout mice (fEPSPs at 50-60 min post-HFS normalized to the pre-stimulation baseline: $151.4 \pm 0.55 \%$ in wild-type slices, $151.8 \pm 0.91 \%$ in CA3-specific Atg5 knockout slices, $151.6 \pm$ $0.81 \%$ in CA1-specific Atg5 knockout slices; one-way ANOVA, $p=0.08$; Fig. 10b). Hence, the inducibility of LTD in adults is restored by Atg5 knockout in postsynaptic neurons. The inputoutput relationship and paired-pulse ratio were comparable in wild-type and CA1-specific Atg5 knockout slices (Fig. 10c, d).

Taken together, these findings indicate that autophagy in the CA1 region is increased in adulthood and that this increase contributes, at least in part, to a low LTD inducibility.

Low LTD inducibility in adults is essential for fear memory. Since LTD is required for learning and memory, we examined the behavior of adult CA1-specific Atg5 knockout mice in which LTD can be easily induced (Fig. 10a). Locomotion (the open field test), anxiety-like behavior (the light/dark box and the elevated zero maze test) were comparable in littermates of $\mathrm{Cre}^{+}$and $\mathrm{Cre}^{-}$mice (Fig. 10e-i). During fear conditioning, $\mathrm{Cre}^{+}$and $\mathrm{Cre}^{-}$mice increased freezing behavior with indistinguishable rates (Fig. 10j). At $24 \mathrm{~h}$ after fear conditioning, although $\mathrm{Cre}^{+}$and $\mathrm{Cre}^{-}$mice had comparable freezing in the cued fear memory test, $\mathrm{Cre}^{+}$mice froze less in the contextual fear memory test (Fig. 10k, l). The specific impairment of contextual but not cued fear memory is consistent with previous reports that contextual, but not cued fear memory, requires the hippocampus ${ }^{55}$.

To test whether memory acquisition is affected in the knockout mice, we measured contextual fear memory at $1 \mathrm{~h}$ after fear conditioning. Knockout and wild-type mice were comparable (Fig. 10m). To test for memory retrieval, we injected the CA1 region with $\mathrm{CQ}$ at $30 \mathrm{~min}$ before the contextual memory test. CQ and vehicle-injected mice had comparable freezing time during the contextual memory test and fear conditioning (Fig. 10n, o). These findings suggest that the knockout mice have deficits in memory consolidation, but not in learning, memory acquisition, or memory retrieval.

To determine the role of increased LTD inducibility in the contextual fear memory impairment of knockout mice, we generated adeno-associated virus (AAV) expressing G2CT, a peptide inhibiting NMDA receptor-dependent GluA2 internalization and $\mathrm{LTD}^{56,57}$. The CA1 region of wild-type littermates of CA1-specific Atg5 knockout mice (8 weeks of age) was injected with $1 \mu$ l virus. Four-week G2CT expression caused less freezing in the contextual fear memory test without changing freezing 
a
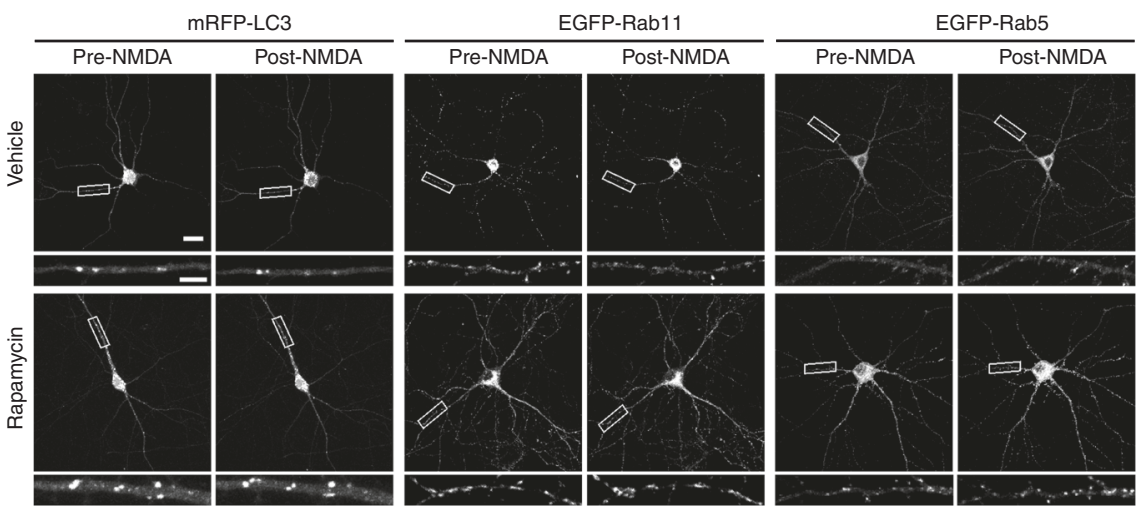

b
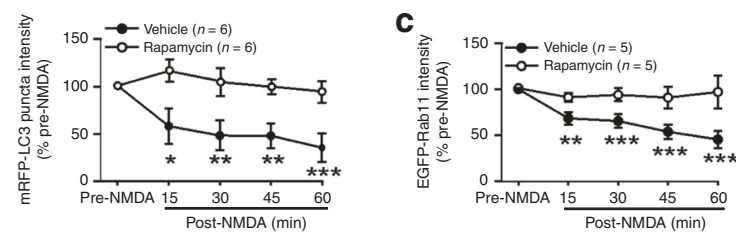

d
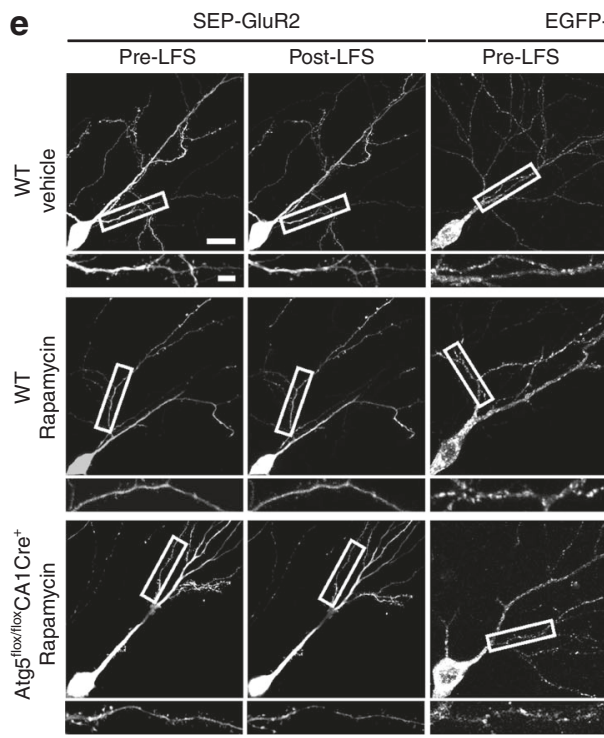

GFP-Rab11

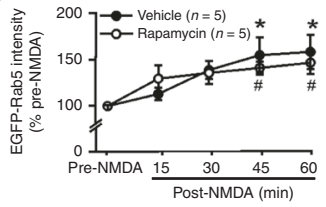

f
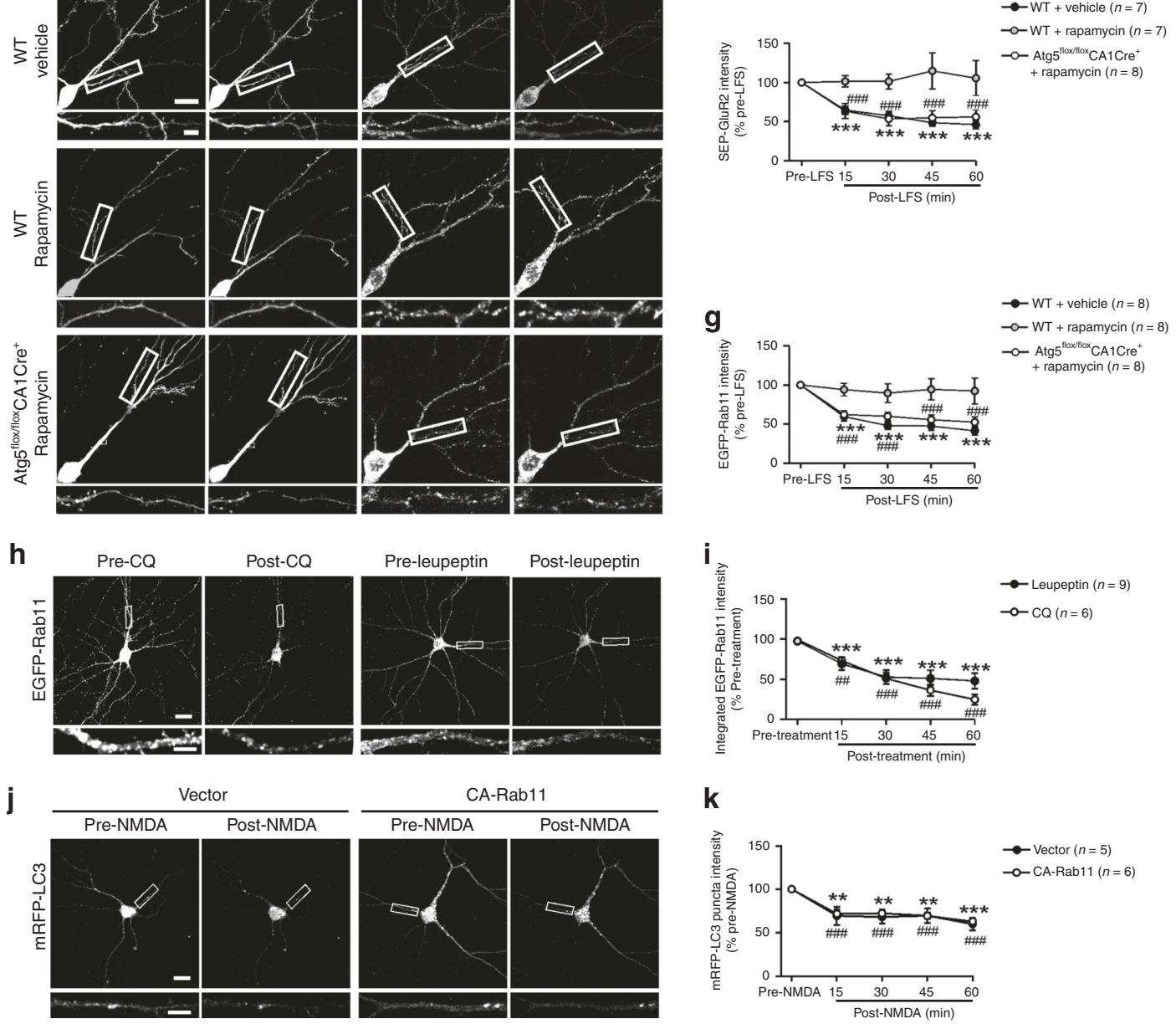

during fear conditioning or cued fear memory test (Supplementary Fig. 8A-C), indicating that G2CT impairs contextual fear memory. LTD was blocked in mice injected with $1 \mu \mathrm{l}$ AAV G2CT (Supplementary Fig. 8D, E). This finding is consistent with previous reports that LTD is required for contextual fear memory ${ }^{5,8}$.
To allow for LTD expression, we injected less virus and shortened viral expression time. Our tests showed that injection of $200 \mathrm{nl}$ virus and 3-week expression decreased but did not abolish LTD and had no significant effect on contextual fear memory (Supplementary Fig. 8D; Fig. 101). The injection of $200 \mathrm{nl}$ scramble virus had no effect on LTD (Supplementary 
Fig. 5 Autophagy inhibition in LTD causes a decrease in recycling endosomes. a Representative images of the same neuron before and after NMDA treatment. b-d Quantification of $\mathbf{a}$; one-way RM ANOVA was used to compare across time points (vehicle: $p=0.001$ in $\mathbf{b}, p=0.000004$ in $\mathbf{c}, p=0.014$ in d; rapamycin: $p=0.199$ in $\mathbf{b}, p=0.927$ in $\mathbf{c}, p=0.006$ in $\mathbf{d}$ ); time points significantly different from the pre-stimulation baseline were identified with Holm-Sidak test; ${ }^{\star} p<0.05,{ }^{\star \star} p<0.01,{ }^{\star \star \star} p<0.001$ for the vehicle group in $\mathbf{b}-\mathbf{d}$; ${ }^{*} p<0.05,{ }^{\# \#} p<0.01$ for the rapamycin group in d. e Representative images of the same neuron in hippocampal slices before and after LFS (low-frequency stimulation). $\mathbf{f}, \mathbf{g}$ Quantification for $\mathbf{e}$; one-way RM ANOVA was used to compare across time points (f: WT + vehicle, $p=4.86 \times 10^{-12} ;$ WT + rapamycin, $p=0.909 ;$ Atg5flox/flox $\mathrm{CA}^{-1 C r e^{+}}+$rapamycin, $p=2 \times 10^{-6}$. g: WT + vehicle, $p=3.87 \times 10^{-10} ; \mathrm{WT}+$ rapamycin, $p=0.900$; Atg $5^{f l o x} /$ flox $C A 1 C r e^{+}+$rapamycin, $\left.p=2.68 \times 10^{-10}\right)$. h, $\mathbf{j}$ Representative images of the same neuron before and after treatment. i Quantification of $\mathbf{h}$; one-way RM ANOVA was used to compare across time points $\left(p=1.0073 \times 10^{-11}\right.$ for CQ and $p=5.85 \times 10^{-7}$ for leupeptin); Holm-Sidak test was used to identify time points significantly different from the pre-stimulation baseline; ${ }^{\star \star \star} p<0.001$ for CQ; $\# \#$ $p<0.001$ for leupeptin. $\mathbf{k}$ Quantification of $\mathbf{j}$; one-way RM ANOVA was used to compare across time points $(p=0.00018$ for vector, $p=0.000003$ for CA-Rab11); Holm-Sidak test was used to identify time points significantly different from pre-stimulation; ${ }^{\star \star \star} p<0.001$ for vectortransfected cells; $\# \# \#$ < 0.001 for CA-Rab11 transfected cells. Data are presented as mean \pm SEM; $n$ indicates the number of cells in $\mathbf{b}-\mathbf{d}$ and $\mathbf{h}-\mathbf{k}$, and the number of slices in $\mathbf{f}$ and $\mathbf{g}$; no adjustments were made for multiple comparisons. Scale bar: $20 \mu \mathrm{m}$ in top and $5 \mu \mathrm{m}$ in lower images. Source data are provided as a Source Data file.

Fig. 8E). $200 \mathrm{nl}$ virus transduced fewer cells than $1 \mu \mathrm{l}$ virus and restored contextual fear memory in $\mathrm{Cre}^{+}$mice without affecting locomotion or anxiety-like behaviors (Fig. 10p-u; Supplementary Fig. 8F). Hence, excessive LTD in CA1-specific Atg5 knockout mice impairs contextual fear memory.

Taken together, these findings indicate that the low inducibility of LTD in adult CA1 neurons is required for the consolidation of contextual fear memory.

\section{Discussion}

Although autophagy has been implicated in neural development and neurotransmitter release, its role in synaptic plasticity is unclear. In this study, we found an unexpected decrease in autophagic flux by NMDA treatment and LFS. This decrease is transient and begins within $15 \mathrm{~min}$ after stimulation. An earlier study reports a delayed increase in autophagy that peaks at $2 \mathrm{~h}$ after NMDA stimulation while no significant changes at earlier times $^{29}$. Different cell culture and stimulation methods may account for the lack of early autophagy changes in this study. The delayed activation of autophagy, however, is not likely to act on the induction or expression of LTD because it occurs long after these processes have started. This notion is consistent with the findings that autophagy inhibitors have no effect on LFS-induced $\mathrm{LTD}^{30}$.

Our results indicate that the early autophagy decrease is both necessary and sufficient for LTD induction. First, we found that rapamycin blocks NMDA receptor-dependent LTD and AMPA receptor internalization. Using Atg5 knockout mice and Atg5 siRNAs, we confirm that rapamycin requires autophagy to take effect. Rapamycin has been shown to inhibit long-lasting LTP (L-LTP) and metabotropic glutamate receptor-dependent LTD (mGluR-LTD) by regulating protein synthesis ${ }^{29,58-61}$. Our study indicates that rapamycin can also influence synaptic plasticity through autophagy. Second, we show that autophagy inhibition induces synaptic depression and AMPA receptor internalization.

In chemical LTD, while rapamycin has no effect on caspase-3 activation, a caspase- 3 inhibitor blocks the decrease in autophagic flux, indicating that autophagy is inhibited by caspase-3, presumably through proteolysis. Autophagy inhibition is crucial for the control of postsynaptic responses by caspase-3, as rapamycin obliterates synaptic depression induced by active caspase- 3 .

What is the specific function of autophagy in NMDAR-LTD? Our study shows that autophagy modifies endocytic recycling. Endocytic recycling is reduced by NMDA receptor activation. This decrease is blocked by rapamycin. Conversely, CQ inhibits endocytic recycling. Consistent with the effect of autophagy on endocytic recycling, NMDA receptor-dependent GluA2 internalization requires autophagy inhibition and CQ promotes
GluA2 internalization. These findings suggest that autophagy contributes to AMPA receptor internalization in NMDAR-LTD by modulating endocytic recycling.

Our study indicates that autophagy regulates recycling endosomes during NMDAR-LTD. This is supported by the finding that during LTD, while rapamycin obliterates the recycling endosome decrease, constitutively active Rab11 does not alter the autophagosome decrease. Additionally, autophagy inhibition is sufficient to reduce recycling endosomes. Hence, it is the reduction of autophagy that attenuates endocytic recycling, but not vice versa. This finding does not contradict the known regulation of autophagy biogenesis by recycling endosomes during such conditions as starvation ${ }^{53}$.

We find that autophagy increases with age and that this increase contributes to reduced LTD inducibility in adulthood. These findings suggest that autophagy serves as a mechanism to dampen LTD in adult brains. It is noted that aging switches the role of autophagy in LTD from an inhibitor to a supporter, as autophagy inhibitors reduce LFS-induced LTD in aged brains ${ }^{30}$. The possible reasons for the switch are that LFS induces tau oligomerization in aged but not in adult, non-aged brains, and autophagy is involved in the degradation of tau oligomers ${ }^{30}$.

NMDAR-LTD is required for various hippocampus-dependent contextual fear memory ${ }^{5,8}$. Contextual fear memory is impaired in adult, CA1-specific Atg5 knockout mice. This is due to increased inducibility of NMDAR-LTD because it can be ameliorated by attenuating NMDAR-LTD with the G2CT peptide. These findings indicate that NMDAR-LTD needs to be fine-tuned to optimize memory.

In sum, this study uncovers unrecognized functions of autophagy in synaptic plasticity, endocytic recycling, the agedependence of LTD induction, and memory consolidation.

\section{Methods}

Animals, DNA constructs, and reagents. The CA3-specific Cre (G32-4) and CA1-specific Cre (T29-1) mice were purchased from the Jackson Laboratory. The Atg $5^{\text {flox }}$ mouse was purchased from RIKEN BioResource Center. All animal procedures followed the US National Institutes of Health Guidelines Using Animals in Intramural Research and were approved by the National Institute of Mental Health Animal Care and Use Committee. The EGFP-Rab5 and EGFP-Rab9 constructs were generously provided by Dr. Juan Bonifacino (National Institute of Child Health and Human Development, National Institutes of Health). The following constructs were purchased from Addgene: EGFP-Rab11a-7 (\#56444), PmRFPLC3B (\#21075), CaMKIIa iChioC 2A tDimer (\#66709), pMXs-puro GFP-p62 (\#38277). The constitutively active Rab11 construct was generated by mutating EGFP-Rab11a-7 [Q(CAA)70L(CTG)]. The Atg5 siRNA (GGCTCACTTTATGT CATGT) and scrambled oligonucleotide (GACGTGAACGGATAACACT) were inserted into the BglII/HindIII site of the pSuper plasmid. cDNAs of HA-Atg5, siRNA-resistant Atg5 (G366C, C372T, T375C, G381C) were inserted into the $\mathrm{XmaI} / \mathrm{KpnI}$ site of the pGW1 vector, HA-Beclin-1 cDNAs were inserted into the $\mathrm{BglII} / \mathrm{EcoR} 1$ site of the pGW1 vector. The sequences encoding the G2CT (KRMKLNINPS, AAG CGG ATG AAG CTG AAC ATC AAC CCT AGC) and the 
a

Vehicle
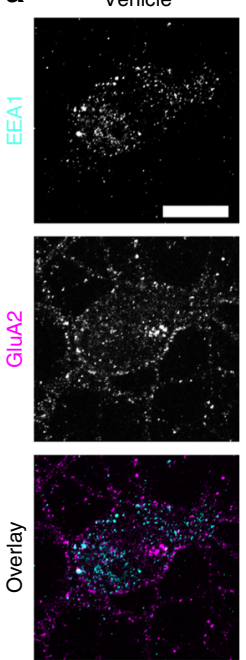

C
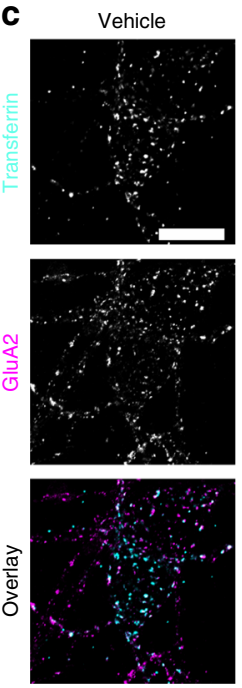

e
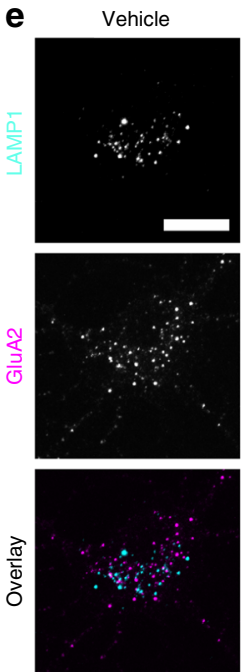

$\mathrm{CQ}$
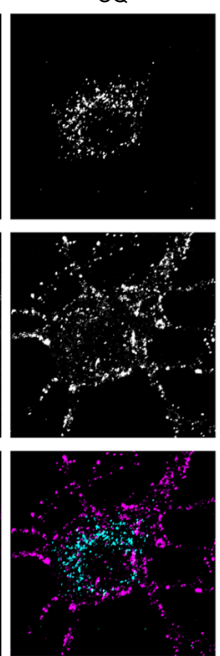

$\mathrm{CQ}$
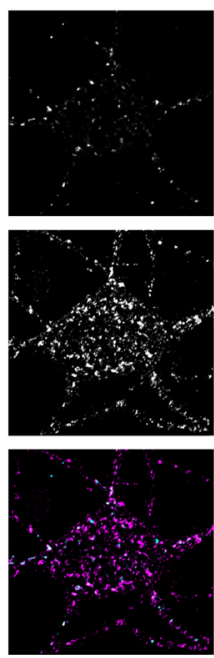

$\mathrm{CQ}$
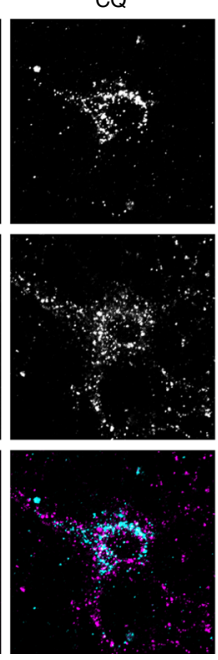

Leupeptin
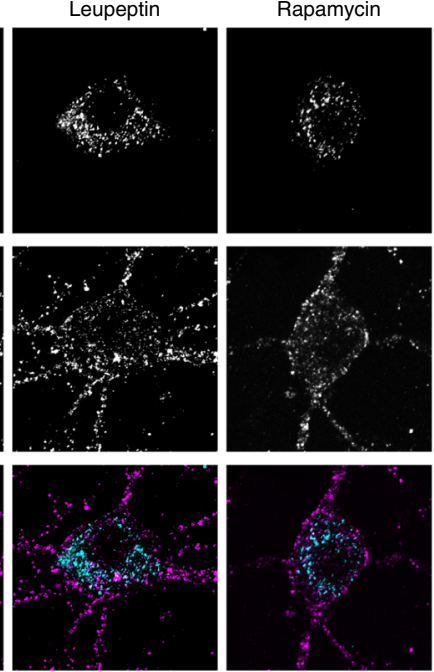

Leupeptin

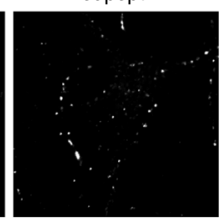

Rapamycin
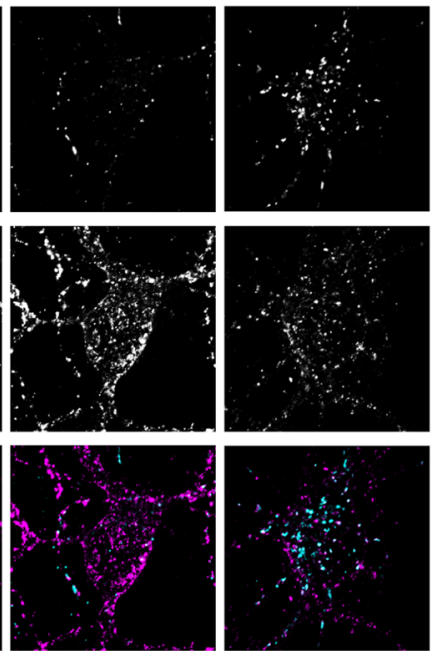

Leupeptin
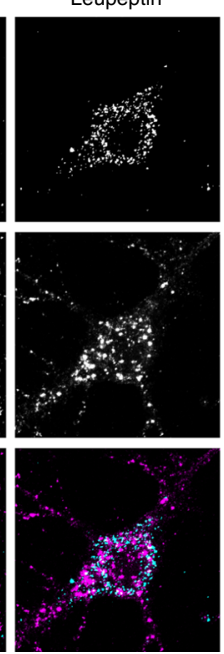
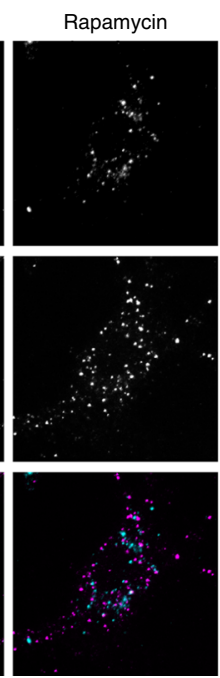

Trehalose
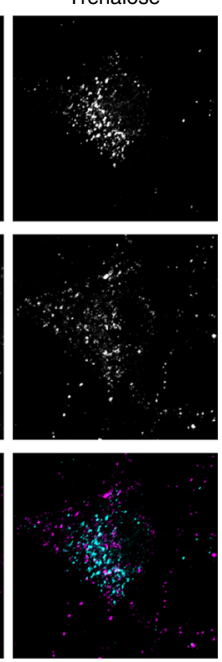

Trehalose
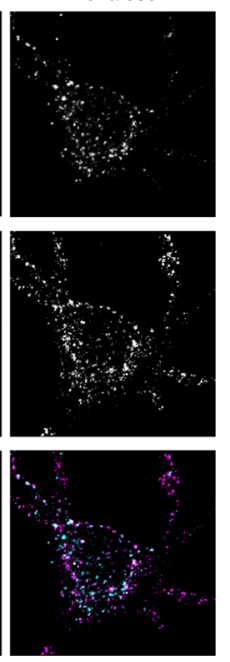

Trehalose
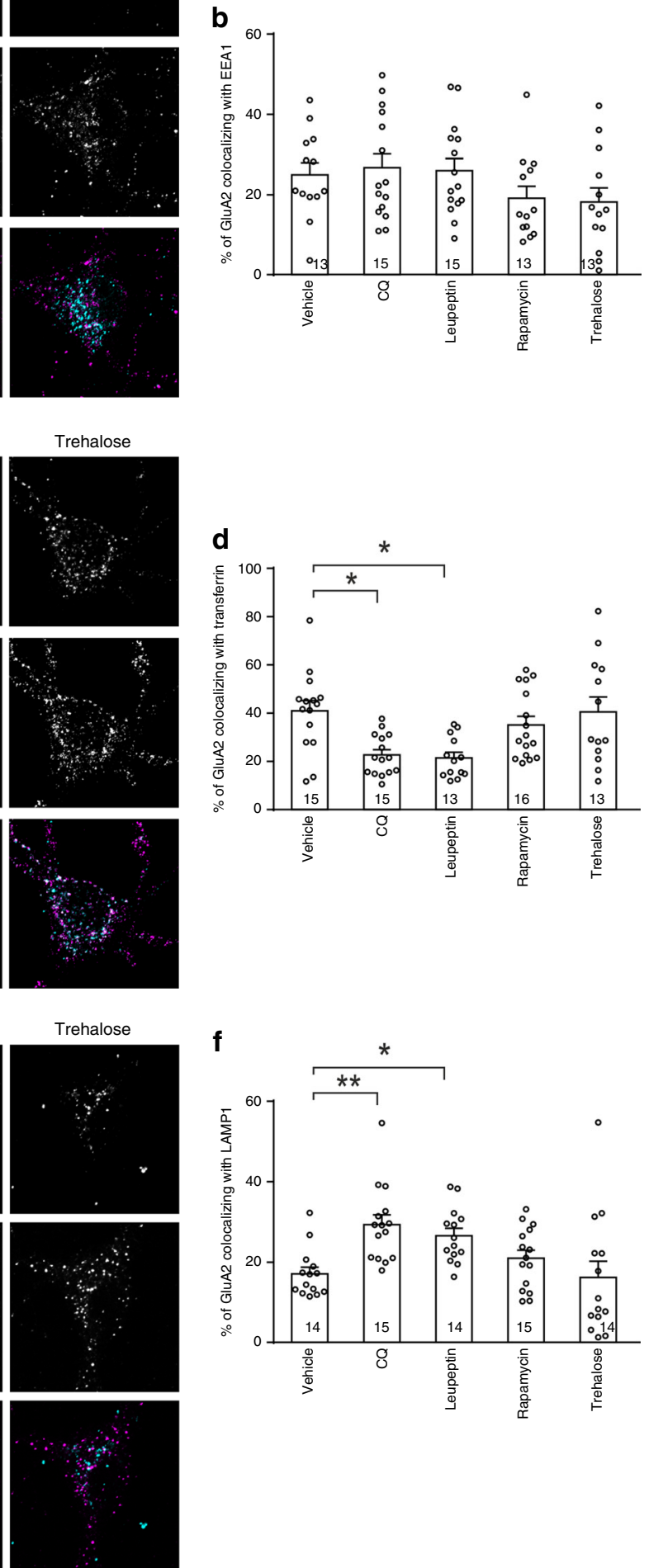

scrambled peptide (RKNNSKMLIP, CGG AAG AAC AGC AAG ATG CTG ATC CCT) were inserted into the EcoR1/Kpnl site of the CaMKIIa iChioC 2A tDimer plasmid. The mRFP-LC3BG120A plasmid was generated by using Phusion Sitedirected Mutagenesis Kit (Thermo Fisher Scientific). The following antibodies were obtained commercially: caspase-3 (1:500 dilution for immunoblotting; Cell Signaling Technology, \#9665), cleaved caspase-3 (1:100 dilution for immunoblotting; Cell Signaling Technology, \#9661), p62 (1:500 dilution for immunoblotting; Cell
Signaling Technology, \#5114), Atg3 (1:500 dilution for immunoblotting; Cell Signaling Technology, \#3415), Atg4B (1:500 dilution for immunoblotting; Cell Signaling Technology, \#5299), Atg5 (11.6 $\mu \mathrm{g} / \mathrm{ml}$ for immunofluorescence; Novus Biologicals, NB110-53818), Atg7 (1:500 dilution for immunoblotting; Cell Signaling Technology, \#2631), Beclin-1 (1:500 dilution for immunofluorescence; Cell Signaling Technology, \#3738), LC3B ( $1 \mu \mathrm{g} / \mathrm{ml}$ for immunoblotting; Novus Biologicals, NB100-2220), actin (1:2000 dilution for immunoblotting; Sigma, A4700), 
Fig. 6 The effect of autophagy activators and inhibitors on endocytic trafficking of GluA2. a, c, e Representative images of primary hippocampal neurons incubated with the GluA2 antibody alone or along with transferrin for $15 \mathrm{~min}$, and then fixed for immunostaining; CQ (120 $\mu \mathrm{M}, 10 \mathrm{~min})$, leupeptin (300 $\mu \mathrm{M}$, $30 \mathrm{~min}$ ), rapamycin $(1 \mu \mathrm{M}, 10 \mathrm{~min})$, or trehalose $(20 \mathrm{mM}, 30 \mathrm{~min})$ were added to the medium during antibody incubation. $\mathbf{b}$ Quantitation for the percentage of GluA2 colocalized with EEA1 in a; Kruskal-Wallis one-way ANOVA on ranks was used to compare across groups $(p=0.131)$. d Quantitation for the percentage of GluA2 colocalized with transferrin in c; Kruskal-Wallis one-way ANOVA on ranks was used to compare across groups ( $p=0.002)$, and Dunn's test was used for post hoc analysis. $\mathbf{f}$ Quantitation for the percentage of GluA2 colocalized with LAMP1 in e; Kruskal-Wallis one-way ANOVA on ranks was used to compare across groups $(p=0.000534)$, and Dunn's test was used for post hoc analysis. Data are presented as mean $\pm \mathrm{SEM}$; ${ }^{\star} p<0.05$, ${ }^{\star \star} p<0.01$; no adjustments were made for multiple comparisons; $n$ in the bar indicates the number of cells in each group. Scale bar, $20 \mu \mathrm{m}$. Source data are provided as a Source Data file.

HA $(1 \mu \mathrm{g} / \mathrm{ml}$ for immunofluorescence; Covance, MMS-101P), $\beta$-galactosidase (6.25 $\mu \mathrm{g} / \mathrm{ml}$ for immunofluorescence; MP Biomedicals, 55976), GluA2 $(10 \mu \mathrm{g} / \mathrm{ml}$ for immunofluorescence; Sigma, MAB397). The following reagents were purchased from Sigma: NMDA, APV, rapamycin, and chloroquine. DEVD-fmk (FMK004) and active caspase-3 (707-C3/CF) were purchased from R\&D systems. Alexa Fluor ${ }^{\mathrm{TM}}$ 555- and Alexa Fluor ${ }^{\mathrm{TM}}$ 488-conjugated transferrin was purchased from Thermo Fisher Scientific. The following reagents were purchased from Cayman: trehalose (\#20517), bafilomycin A1 (\#11038), and leupeptin (\#14026). AA2 was purchased from Enzolife Sciences (ALX-420-085). The sequences of primers used to generate DNA constructs are listed in the following table.

\begin{tabular}{|c|c|c|c|}
\hline Plasmid Name & Vector & $\begin{array}{l}\text { Restriction } \\
\text { Site }\end{array}$ & Forward $(F)$ and Reverse primers $(R)$ \\
\hline \multirow[t]{2}{*}{$\begin{array}{l}\text { EGFP-CA- } \\
\text { Rab11 }\end{array}$} & \multirow[t]{2}{*}{ Addgene\#56444 } & \multirow[t]{2}{*}{$\begin{array}{l}\text { Site-directed } \\
\text { mutagenesis }\end{array}$} & $\begin{array}{l}\text { F - CAGCAGGGCTAGAGCGATATCGAGC } \\
\text { TATAACATCAGCATATTATC } \mathbf{R} \text { - GCTC }\end{array}$ \\
\hline & & & $\begin{array}{l}\text { GATATCGCTCTAGCCCTGCTGTGTCCCATA } \\
\text { TCTGTG }\end{array}$ \\
\hline \multirow[t]{3}{*}{ Atg5 siRNA } & \multirow[t]{3}{*}{ pSuper } & \multirow[t]{3}{*}{ BglII/HindIII } & F - GGAAGATCTGGCTCACTTTATGTCA \\
\hline & & & TGTAAGCTTGGG R -CCCAAGCTTACAT \\
\hline & & & GACATAAAGTGAGCCAGATCTTCC \\
\hline \multirow{2}{*}{$\begin{array}{l}\text { Scrambled } \\
\text { oligonucleotide }\end{array}$} & \multirow[t]{2}{*}{ pSuper } & \multirow[t]{2}{*}{ BglII/HindIII } & F - GGAAGATCTGACGTGAACGGATAA \\
\hline & & & $\begin{array}{l}\text { CACTAAGCTTGGG R -CCCAAGCTTAG } \\
\text { TGTTATCCGTTCACGTCAGATCTTCC }\end{array}$ \\
\hline \multirow[t]{3}{*}{ HA-Atg5 } & \multirow[t]{3}{*}{ pGW1 } & \multirow[t]{3}{*}{$\mathrm{XmaI} / \mathrm{KpnI}$} & F - TCCCCCCGGGATGTGTGGTTTGGAC \\
\hline & & & GAATTCCAACTTGTT R - CGGGGTACC \\
\hline & & & $\begin{array}{l}\text { TGAGCAGCGTAATCTGGAACGTCATA } \\
\text { TGGAT }\end{array}$ \\
\hline \multirow{2}{*}{$\begin{array}{l}\text { siRNA- } \\
\text { resistant Atg5 }\end{array}$} & \multirow[t]{2}{*}{ Atg5-siRNA } & \multirow[t]{2}{*}{ BglII/HindIII } & F - GGAAGATCTCGCTCATTTCATGTC \\
\hline & & & $\begin{array}{l}\text { ATCTAAGCTTGGG R - CCCAAGCTTCC } \\
\text { CAAGCTTAGATGACATGAAATGAGCG }\end{array}$ \\
\hline \multirow[t]{4}{*}{ HA-Beclin-1 } & \multirow[t]{4}{*}{ pGW1 } & \multirow[t]{3}{*}{ BglII/EcoRI } & F - CGCGGATCCATGGAAGGGTCTAAG \\
\hline & & & ACGTCCAACAACAG R - CCGGAATTCA \\
\hline & & & GCGTAATCTGGAACATCGTATGGGTAG \\
\hline & & \multirow[t]{3}{*}{ EcoRI/Kpnl } & $\begin{array}{l}\text { CGG } \\
\text { F- CCGGAATTCAAGCGGATGAAGCTGA }\end{array}$ \\
\hline \multirow{2}{*}{ G2CT } & \multirow{2}{*}{ Addgene, \#66709 } & & ACATCAACCCTAGC R - CGGGGTACCGC \\
\hline & & & TAGGGTTGATGTTCAGCTTCATCCGCTT \\
\hline \multirow{3}{*}{$\begin{array}{l}\text { Scrambled } \\
\text { peptide }\end{array}$} & \multirow{3}{*}{ Addgene, \#66709 } & \multirow{3}{*}{ EcoRI/Kpnl } & F- CCGGAATTCCGGAAGAACAGCAAG \\
\hline & & & ATGCTGATCCCT R - CGGGGTACCAG \\
\hline & & & GGATCAGCATCTTGCTGTTCTTCCG \\
\hline \multirow[t]{2}{*}{ mRFP-LC3 } & \multirow[t]{2}{*}{ pGW1 } & \multirow[t]{2}{*}{$\mathrm{KpnI} / \mathrm{EcoRI}$} & F - CGGGGTACCTCATGGCCTCCTCCG \\
\hline & & & $\begin{array}{l}\text { AGGACGTCATC R - GCGCGAATTCTCA } \\
\text { CAAGCATGGCTCTC }\end{array}$ \\
\hline \multirow{3}{*}{$\begin{array}{l}\text { mRFP- } \\
\text { LC3BG120A }\end{array}$} & \multirow{3}{*}{$\begin{array}{l}\text { pGW1-mRFP- } \\
\text { LC3 }\end{array}$} & \multirow{3}{*}{$\begin{array}{l}\text { Site-directed } \\
\text { mutagenesis }\end{array}$} & F - CCCAGGAGACGTTCGCGACAGCACT \\
\hline & & & GGCTGTTACATAC R - CCAGTGCTGTC \\
\hline & & & GCGAACGTCTCCTGGGAGGCATAGACC \\
\hline
\end{tabular}

Surgery. 7-week-old male mice were anesthetized by intraperitoneal injection of Ketamine/Xylazine (Ketamine: $100 \mathrm{mg} / \mathrm{kg}$; Xylazine: $8 \mathrm{mg} / \mathrm{kg}$ ). A bilateral craniotomy was made above the hippocampus. $200 \mathrm{nl}$ or $1 \mu \mathrm{l} \mathrm{AAV}$ virus was injected into the hippocampal CA1 region (AP: $2.0 \mathrm{~mm}, \mathrm{ML}: \pm 1.5 \mathrm{~mm}, \mathrm{DV}:-1.5 \mathrm{~mm}$ ) with a $5 \mu \mathrm{l}$ gas-tight syringe (Hamilton, \#87931, \#7803-05) at a speed of $100 \mathrm{nl} / \mathrm{min}$.

Hippocampal slice culture. 6- to 8-day-old male C57BL/6 mice were decapitated, and the brain was placed immediately in cold cutting solution composed of (in $\mathrm{mM}): 238$ sucrose, $2.5 \mathrm{KCl}, 26 \mathrm{NaHCO}_{3}, 1 \mathrm{NaH}_{2} \mathrm{PO}_{4}, 5 \mathrm{MgCl}_{2}, 11 \mathrm{D}$-glucose and $1 \mathrm{CaCl}_{2}$. Hippocampal slices $(350 \mu \mathrm{m})$ were cut with a vibratome (Leica, VT1000s) and placed on semi-permeable membrane inserts (Millipore, R7AA72342) in a sixwell plate containing culture medium (78.8\% minimum essential medium, $20 \%$ heat-inactivated horse serum, $25 \mathrm{mM}$ HEPES, $10 \mathrm{mM}$ D-glucose, $26 \mathrm{mM} \mathrm{NaHCO}_{3}$, $2 \mathrm{mM} \mathrm{CaCl} 2,2 \mathrm{mM} \mathrm{MgSO} 4,0.0012 \%$ ascorbic acid, $1 \mu \mathrm{g} / \mathrm{ml}$ insulin; $\mathrm{pH} 7.3$; $320-330 \mathrm{mOsm})$. No antibiotics were added and the medium was replaced every 2 days. Slices were biolistically transfected using a gene gun (Bio-Rad, Helios Genegun system) at 5-6 days in vitro and used for electrophysiology at 3 days after transfection

Acute hippocampal slice. Mice (16-19 days of age, male) were anesthetized with isoflurane and decapitated. The brain was rapidly removed and chilled in ice-cold sucrose solution containing the following (in $\mathrm{mM}$ ): $2.5 \mathrm{KCl}, 26 \mathrm{NaHCO}_{3}, 1.25$ $\mathrm{NaH}_{2} \mathrm{PO}_{4}, 185$ sucrose, 25 D-glucose, 20 HEPES, 5 sodium ascorbate, 2 thiourea,
3 sodium pyruvate, $0.5 \mathrm{CaCl}_{2}$, and $10 \mathrm{MgSO}_{4}, \mathrm{pH}$ 7.3. Transverse hippocampal slices ( $350 \mu \mathrm{m}$ thick) were cut in ice-cold sucrose solution using a vibratome (VT$1000 \mathrm{~s}$, Leica). The hippocampal slices were incubated in warm $\left(32^{\circ} \mathrm{C}\right)$ ACSF solution (in mM; $124 \mathrm{NaCl}, 2.5 \mathrm{KCl}, 1.2 \mathrm{NaH}_{2} \mathrm{PO}_{4}, 24 \mathrm{NaHCO}_{3}, 5$ HEPES, $12.5 \mathrm{D}$ glucose, $2 \mathrm{MgCl}_{2}$ and $\mathrm{CaCl}_{2} \mathrm{pH}$ 7.3) for $30 \mathrm{~min}$ and then allowed to cool down to room temperature for $30 \mathrm{~min}$ before being transferred to the recording chamber. All solutions were continuously bubbled with $95 \% \mathrm{O}_{2} / 5 \% \mathrm{CO}_{2}$.

Electrophysiology. Hippocampal slices were perfused with ACSF at $2 \mathrm{ml} / \mathrm{min}$. The stimulating electrode was placed on the Schaffer collateral pathway. For field recordings, recording pipettes $(1-2 \mathrm{M} \Omega)$ were filled with the bath solution and placed in the CA1 region. LTD was induced with low-frequency stimulations (900 pulses, $1 \mathrm{~Hz}, 15 \mathrm{~min})$, and LTP was induced with high-frequency stimulations (2 trains of 100 pulses at $100 \mathrm{~Hz}$ with an inter-train interval of $15 \mathrm{~s}$ ). For whole-cell recordings, the patch pipette (4-6 M $\Omega$ ) solution is composed of (in $\mathrm{mM}$ ): 130.0 cesium methanesulfonate, $8.0 \mathrm{NaCl}, 4.0 \mathrm{Mg}$-ATP, $0.3 \mathrm{Na}$-GTP, 0.5 EGTA, 10.0 HEPES and 5.0 QX-314 at pH 7.3. EPSCs of CA1 pyramidal neurons were recorded at a holding potential of $-70 \mathrm{mV}$. The series resistance and input resistance were monitored on-line and analyzed with the Clampex program off-line. Only cells with a series resistance of $<25 \mathrm{M} \Omega$ and a $<10 \%$ drift in both series resistance and input resistance during the recording period were included.

Neural culture and transfection. Primary hippocampal neurons were prepared from embryonic day (E) 18-19 rat, male and female embryos. Neurons were seeded on coverslips or culture plates coated with poly-D-lysine $(20 \mu \mathrm{g} / \mathrm{ml})$ and laminin $(1.25 \mu \mathrm{g} / \mathrm{ml})$ at a density of $\sim 750$ cells $/ \mathrm{mm}^{2}$. Neurons were cultured in Neurobasal medium supplemented with 2\% B27 and 1\% GlutaMax (Thermo Fisher) and transfected with Lipofectamine ${ }^{\mathrm{TM}} 2000$ (Thermo Fisher) following the manufacturer's instructions.

GluA2 internalization assay. Neurons (DIV 17) were treated with TTX ( $1 \mu \mathrm{M})$ for $30 \mathrm{~min}$, followed by incubation with an antibody against the $\mathrm{N}$-terminus of GluA2 $(10 \mu \mathrm{g} / \mathrm{ml})$ for $15 \mathrm{~min}$ at $37^{\circ} \mathrm{C}$ and then NMDA stimulation $(30 \mu \mathrm{M}, 5 \mathrm{~min})$ or sham treatment. Neurons were fixed with $4 \%$ formaldehyde in PBS containing $4 \%$ sucrose at $10 \mathrm{~min}$ after stimulation. Surface-remaining GluA2 was labeled by incubation with Alexa Fluor ${ }^{\circledR} 555$-conjugated secondary antibodies (Thermo Fisher). Internalized GluA2 was stained with Alexa Fluor ${ }^{\circledR} 488$-conjugated secondary antibodies (Thermo Fisher) following permeabilization with methanol $\left(-20^{\circ} \mathrm{C}, 1 \mathrm{~min}\right)$.

Transferrin uptake assay. Cultured hippocampal neurons (DIV 17) were washed with warm DMEM $\left(37^{\circ} \mathrm{C}\right)$ for three times before incubation with Alexa Fluor ${ }^{\mathrm{TM}}$ 555 (for cells transfected with the constitutively active EGFP-Rab11 plasmid)or Alexa Fluor ${ }^{\mathrm{TM}} 488$-conjugated transferrin $(25 \mu \mathrm{g} / \mathrm{ml}$ in Neurobasal medium) at $37^{\circ} \mathrm{C}$ for 15 min Following wash with warm DMEM for three times to remove surface transferrin, neurons were fixed with $4 \%$ formaldehyde (in PBS containing $4 \%$ sucrose) for imaging acquisition of constitutively active EGFP-Rab11 expressing cells or incubated with the $\beta$-galactose antibody (for cells transfected with the $\beta$-galactose antibody; $2 \mathrm{~h}$, room temperature) and then with Alexa Fluor ${ }^{\circledR} 488$ conjugated secondary antibody $(1 \mathrm{~h}$, room temperature).

Immunostaining. Neurons grown on coverslips were fixed with $4 \%$ formaldehyde (in PBS containing $4 \%$ sucrose) for $15 \mathrm{~min}$ at room temperature. Following wash, neurons were incubated with primary antibodies in GDB buffer $\left(12 \mathrm{mM} \mathrm{Na}_{2} \mathrm{HPO}_{4}\right.$ $48 \mathrm{mM} \mathrm{NaH}_{2} \mathrm{PO}_{4}, 163 \mathrm{mM} \mathrm{NaCl}, 1 \%$ Triton $\mathrm{X}-100$ and $0.36 \%$ gelatin at $\mathrm{pH} 7.4$ ) overnight at $4{ }^{\circ} \mathrm{C}$, washed with PBS, incubated with secondary antibodies in GDB buffer for $1 \mathrm{~h}$ at room temperature, washed with PBS, and then mounted on slides with mounting media. For immunohistochemistry in brain sections, P19 mice deeply anesthetized with isoflurane were transcardially perfused with $4 \%$ formaldehyde in PBS. Brains were removed, fixed for an additional $12 \mathrm{~h}$ with $4 \%$ formaldehyde in PBS, and then immersed in 30\% sucrose in PBS followed by cutting into $30 \mu \mathrm{m}$ cryosections with a Leica CM 3050 s cryostat. Brain sections were blocked with $5 \%$ horse serum in PBST (PBS with $0.1 \%$ Tween 20 ) followed by incubation with an antibody against Atg5 (Novus, \#NB110-53818, 1:500 dilution) 
a

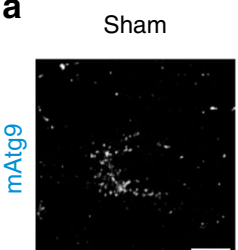

Rapamycin

Rapamycin

$\mathrm{CQ}$

Leupeptin

Bafilomycin A1

NMDA
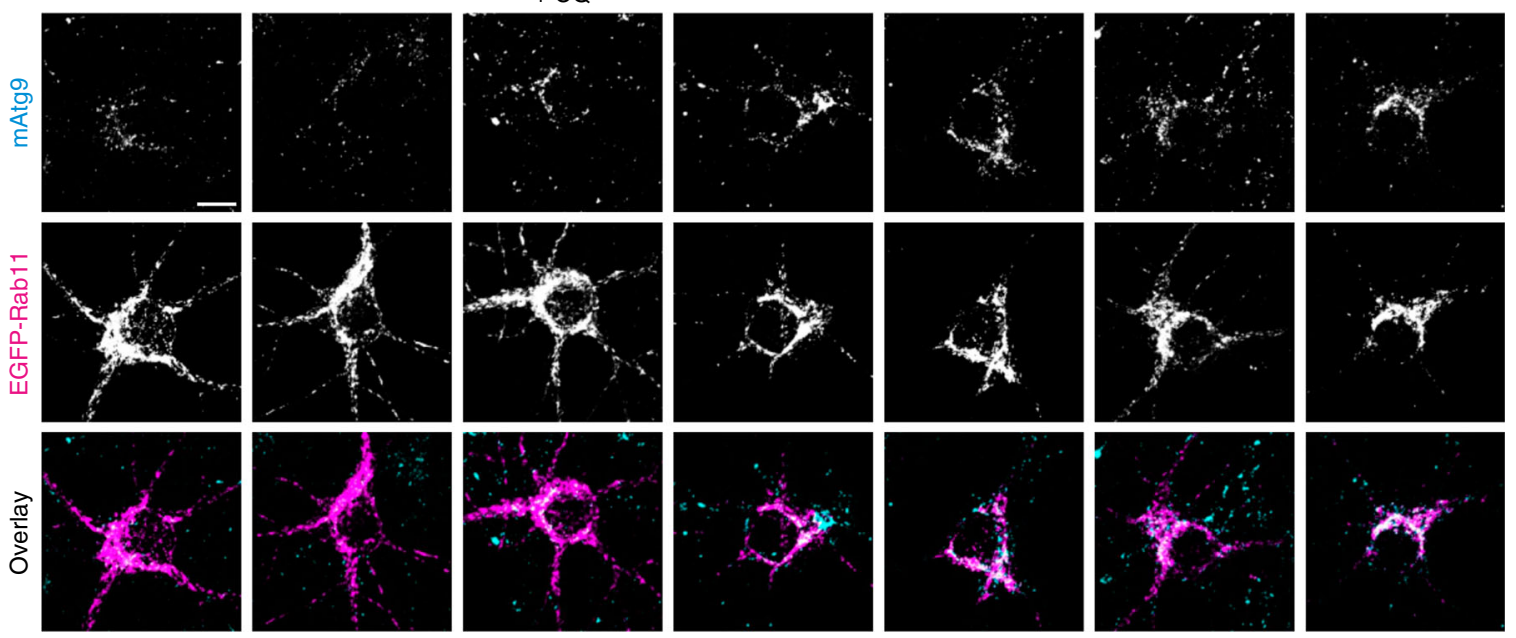

b

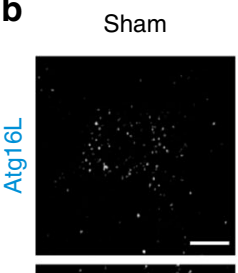

Rapamycin

Rapamycin

$\mathrm{CQ}$

Leupeptin

Bafilomycin A1

NMDA
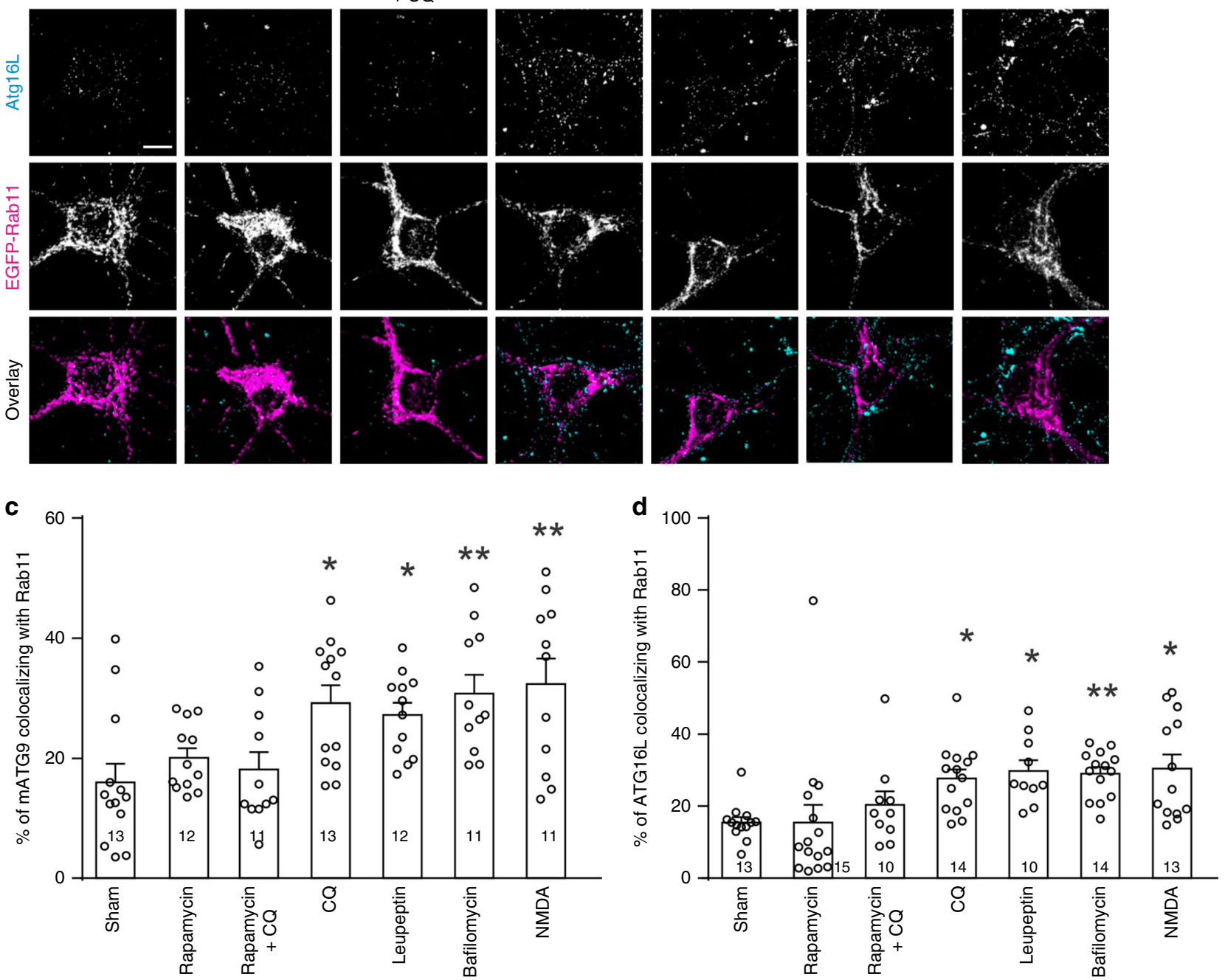

Fig. 7 Autophagy inhibition leads to an increase in Atg16L and Atg9 in recycling endosomes. a, b Representative images of primary hippocampal neurons treated with rapamycin ( $1 \mu \mathrm{M}, 10 \mathrm{~min})$, leupeptin $(300 \mu \mathrm{M}, 30 \mathrm{~min})$, bafilomycin A1 (20 $\mu \mathrm{M}, 30 \mathrm{~min}), \mathrm{NMDA}(30 \mu \mathrm{M}, 5 \mathrm{~min}), \mathrm{CQ}(120 \mu \mathrm{M}, 10 \mathrm{~min})$, or CQ along with rapamycin ( $1 \mu \mathrm{M}$, pretreated for $20 \mathrm{~min})$; scale bar, $10 \mu \mathrm{m}$. c Quantitation of the percentage of mAtg9 colocalized with Rab11 in a; one-way ANOVA was used to compare across groups ( $p=0.000186$ ), and Student-Newman-Keuls test was used to identify groups significantly different from the sham group. d Quantitation of the percentage of Atg16L colocalized with Rab11 in c; Kruskal-Wallis one-way ANOVA on ranks was used to compare across groups ( $p=5 \times$ $\left.10^{-6}\right)$, and Dunn's test was used to identify groups significantly different from the sham group. Data are presented as mean \pm SEM; ${ }^{\star} p<0.05,{ }^{\star \star} p<0.01 ;$ no adjustments were made for multiple comparisons; $n$ in the bar indicates the number of cells in each group. Source data are provided as a Source Data file. 
a

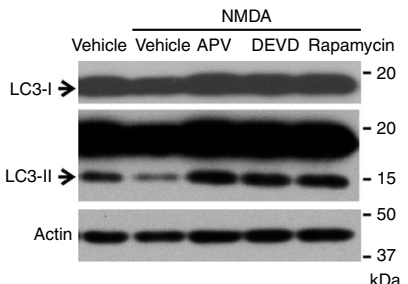

b

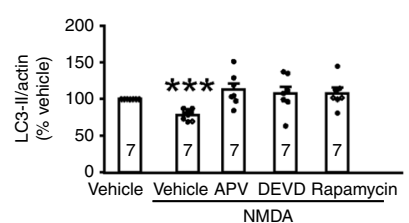

c
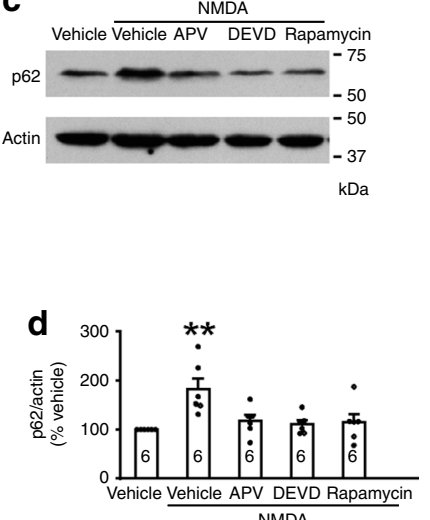
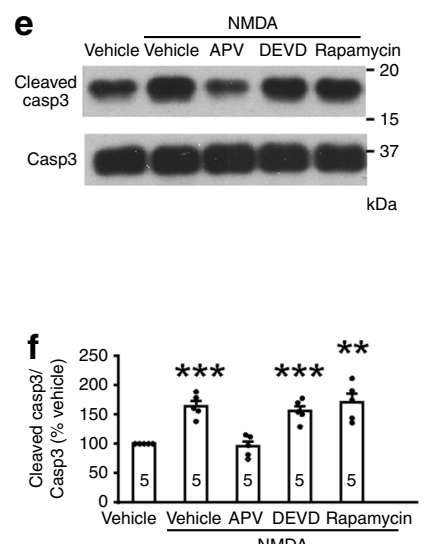

g

h
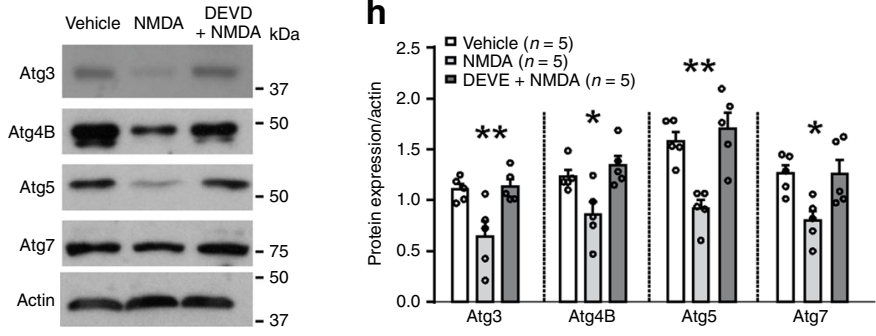

i
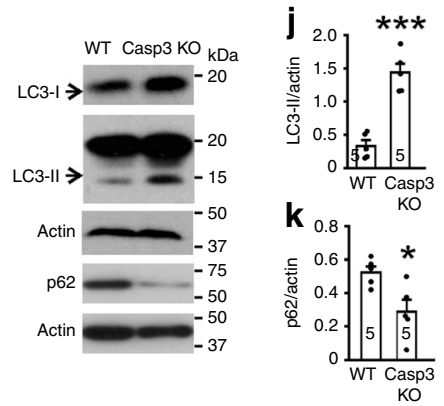

m
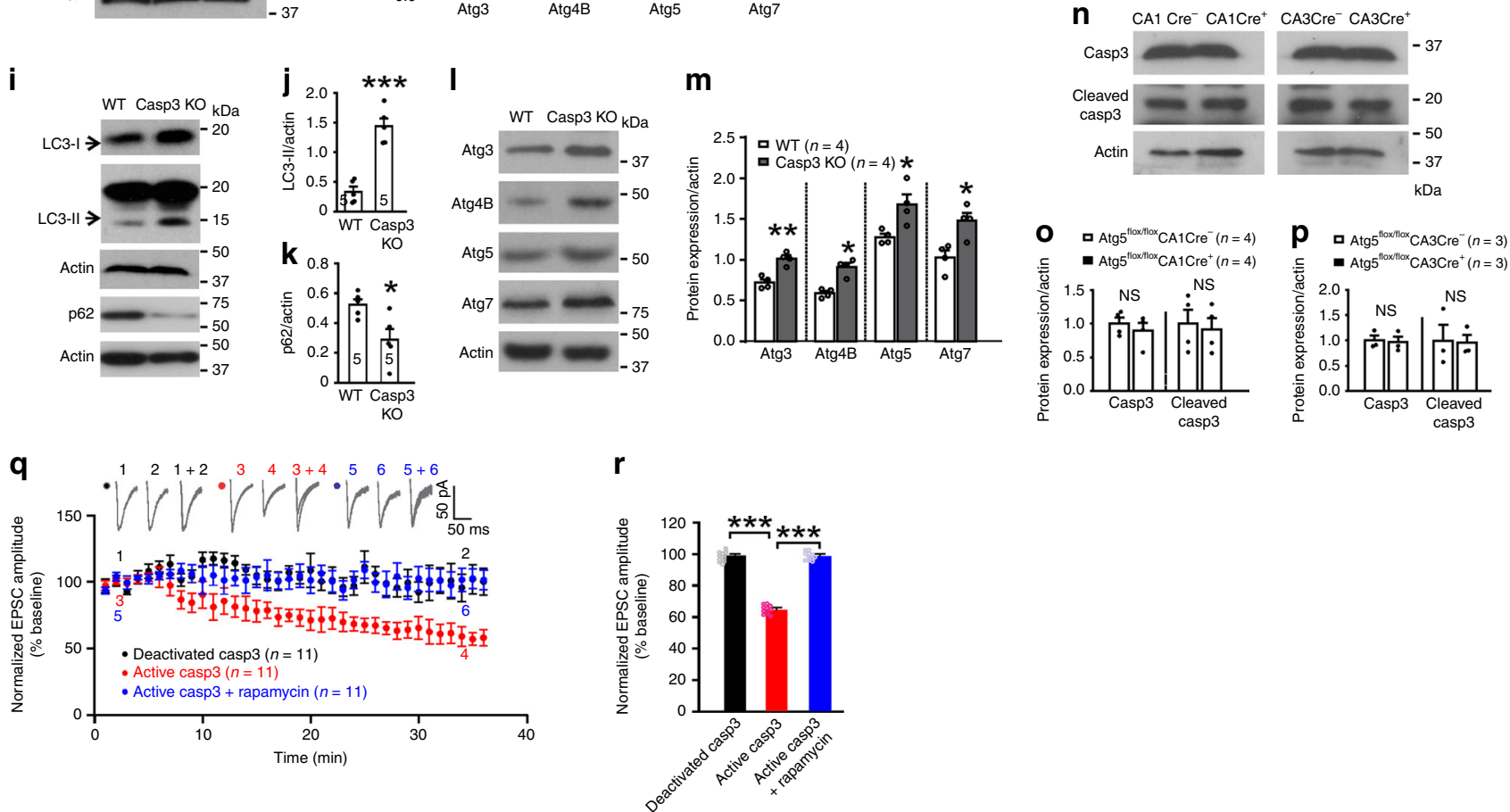

Fig. 8 Autophagy inhibition in LTD requires caspase-3 activity. a, c, e Representative blots of primary hippocampal neurons lysed 15 min after NMDA treatment; DEVD $(5 \mu \mathrm{M})$, APV $(100 \mu \mathrm{M})$, rapamycin $(1 \mu \mathrm{M}), 5$ min pretreatment. b Quantification for a. d Quantification for $\mathbf{c} . \mathbf{f}$ Quantification for e. b, d, f Kruskal-Wallis one-way ANOVA on ranks was used for statistical analysis ( $p=0.009$ in $\mathbf{b}, p=0.009$ in $\mathbf{d}, p=0.001$ in $\mathbf{f}$ ), and Student-Neuman-Keuls test for comparison with vehicle. $\mathbf{g}$ Representative blot of primary hippocampal neurons lysed 30 min after NMDA treatment. $\mathbf{h}$ Quantification for g; one-way ANOVA was used for statistical analysis ( $p=0.007$ for Atg3, $p=0.012$ for Atg4B, $p=0.000847$ for Atg5, $p=0.017$ for Atg7), and Student-Neuman-Keuls test for comparison with vehicle. i, I Representative immunoblots of the CA1 region (P17-19). j Quantification of LC3-II in $\mathbf{i}(p=0.000186)$. $\mathbf{k}$ Quantification of $p 62$ in $\mathbf{i}(p=0.0327)$. $\mathbf{m}$ Quantification for I ( $p=0.00889$ for Atg3, $p=0.0203$ for Atg $4 \mathrm{~B}, p=0.0323$ for Atg5, $p=0.0275$ for Atg7); two-tailed pared t-test was used for statistical analysis in $\mathbf{j}, \mathbf{k}$, and $\mathbf{m}$. $\mathbf{n}$ Representative blot of the CA1 region. $\mathbf{o}$ Quantification for the CA1 lysate in $\mathbf{n} . \mathbf{p}$ Quantification for the CA3 lysate in $\mathbf{n}$. Two-tailed paired t-test or Wilcoxon Signed Rank Test was used for full-length caspase-3 $(p=0.479$ in $\mathbf{0}, p=0.867$ in $\mathbf{p})$ and cleaved caspase-3 ( $p=0.375$ in $\mathbf{o}, p=0.806$ in $\mathbf{p})$. $\mathbf{q}, \mathbf{r}$ Whole-cell recordings of CA1 neurons; caspase-3 ( 2 ng/ $\mu$ l), rapamycin $(10 \mu \mathrm{M})$, deactivated caspase-3 $(2 \mathrm{ng} / \mu \mathrm{l})$. (R) EPSCs at 30-35 min were normalized to the 0-5 min baseline; one-way ANOVA was used to compare across groups $\left(p=3.6 \times 10^{-18}\right)$, and Bonferroni test for post hoc analysis. The number in the bars and $n$ represent the number of cell cultures $(\mathbf{b}, \mathbf{d}, \mathbf{f}, \mathbf{h})$, animals $(\mathbf{j}, \mathbf{k}, \mathbf{m}, \mathbf{o}, \mathbf{p})$, or cells $(\mathbf{q})$; no adjustments for multiple comparisons. Data show mean \pm SEM. ${ }^{\star} p<0.05,{ }^{\star \star} p<0.01,{ }^{\star \star *} p<0.001$. Source data are provided as a Source Data file. 

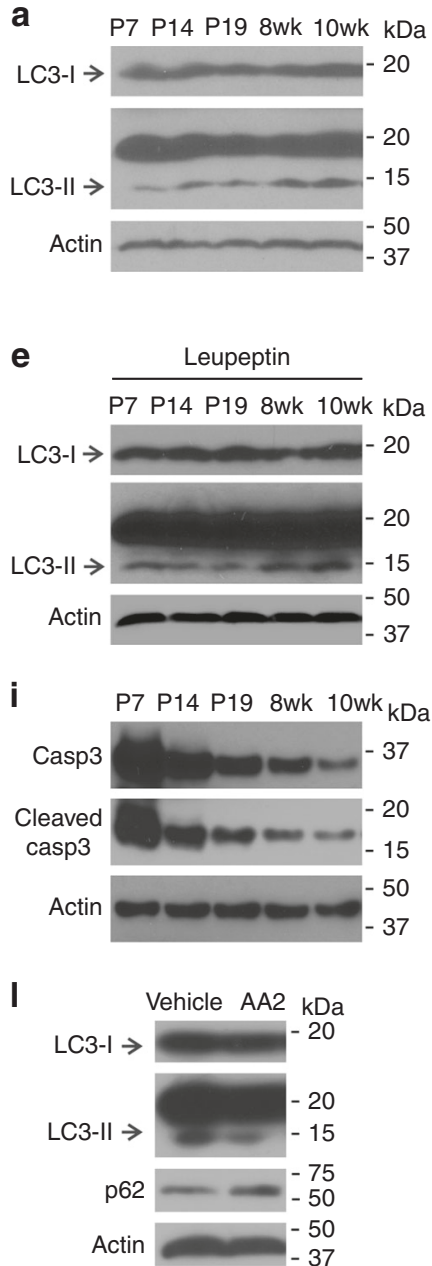

b

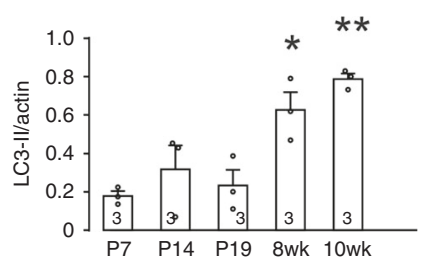

f

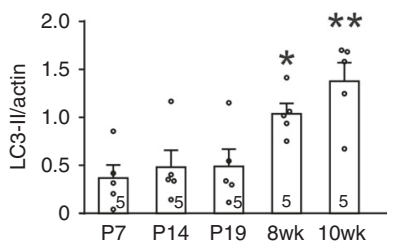

j

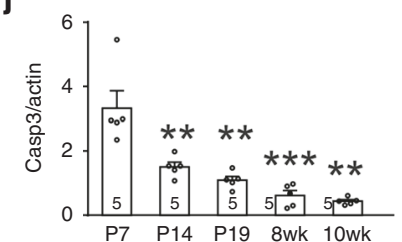

m

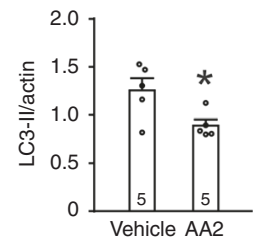

c

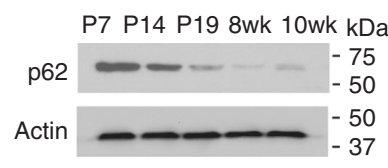

g

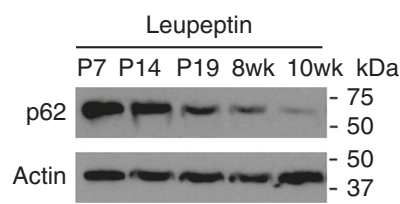

d

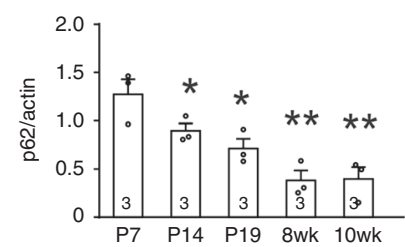

h

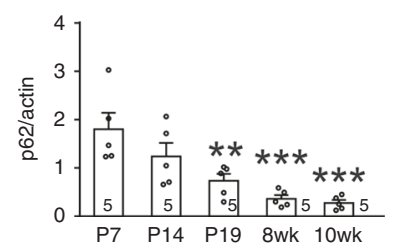

k

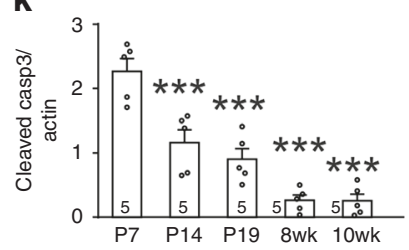

n

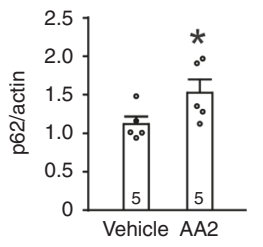

Fig. 9 Autophagy increases and caspase-3 decreases in the adult CA1 region. $\mathbf{a}, \mathbf{c}, \mathbf{e}, \mathbf{g}, \mathbf{i}$, I Representative blots of the CA1 region from acute hippocampal slices. b Quantification for $\mathbf{a}$; one-way ANOVA was used to compare across ages ( $p=0.001)$, and Student-Newman-Keuls test was used to identify ages significantly different from postnatal day 7 (P7). d Quantification for c; one-way ANOVA was used to compare across ages ( $p=0.001$ ), and Student-Newman-Keuls test was used to identify ages significantly different from P7. f Quantification for e; one-way ANOVA was used to compare across ages $(p=0.000903)$, and Student-Newman-Keuls test was used to identify ages significantly different from P7. $\mathbf{h}$ Quantification for $\mathbf{g}$; one-way ANOVA was used to compare across groups $(p=0.000229)$, and Student-Newman-Keuls test was used to identify ages significantly different from P7. j, k Quantification for $\mathbf{i}$; Kruskal-Wallis one-way ANOVA on ranks or one-way ANOVA was used to compare across groups ( $p=0.000347$ for $\mathbf{j}, p=7.6141 \times 10^{-8}$ for $\mathbf{k}$ ), and Student-Newman-Keuls test was used to identify ages significantly different from P7. I Representative blots of the CA1 region of adult wild-type mice (10-weeks of age) treated with AA2 $(20 \mu \mathrm{M})$ or vehicle. $(\mathbf{m}, \mathbf{n})$ Quantification for $\mathbf{l}$; two-tailed paired t-test was used for statistical analysis ( $p=0.0292$ for $\mathbf{m}, p=0.0202$ for $\mathbf{n}$ ). The number in the bars and $n$ represent the number of animals; no adjustments were made for multiple comparisons. Data show mean \pm SEM. ${ }^{\star} p<0.05,{ }^{\star \star} p<0.01,{ }^{\star \star \star} p<0.001$. Source data are provided as a Source Data file.

overnight at $4{ }^{\circ} \mathrm{C}$. After primary antibody incubation, the sections were washed and incubated with an anti-rabbit, HRP-conjugated secondary antibody (Cell signaling, $\# 7074,1: 500$ dilution) at room temperature for $1 \mathrm{~h}$. The sections were washed and developed using TSA plus reagent (PerkinElmer, \#NEL741E001KT), then counterstained with DAPI and mounted on slides.

Image acquisition. For time-lapse imaging, cultured hippocampal slices and primary hippocampal neurons were transferred to a chamber mounted on the sample stage of an Olympus BX61WI confocal microscope, and perfused with artificial cerebrospinal fluid (ACSF) composed of (in mM): $124.0 \mathrm{NaCl}, 2.5 \mathrm{KCl}, 24.0 \mathrm{NaHCO}_{3}, 1.2$ $\mathrm{NaH}_{2} \mathrm{PO}_{4}, 2.0 \mathrm{CaCl}_{2}, 2.0 \mathrm{MgSO}_{4}$, $12.5 \mathrm{D}$-glucose, 5.0 HEPES (pH 7.4, bubbled with $95 \% \mathrm{O}_{2} / 5 \% \mathrm{CO}_{2}$ ) at $2 \mathrm{ml} / \mathrm{min}$. Images were acquired with a $60 \mathrm{X}$ objective (NA 1.0 ). Cultured hippocampal slices were stimulated by placing the electrode on the Schaffer collateral pathway near transfected CA1 neurons with low-frequency stimulations (900 pulses, $1 \mathrm{~Hz}$ for LTD induction) or high-frequency stimulations (two trains of 100 pulses at $100 \mathrm{~Hz}$ with an inter-train interval of $15 \mathrm{~s}$ for LTP induction). Images of fixed hippocampal neurons were acquired using a Zeiss LSM780 confocal microscope with a 63X (NA 1.4) objective. Fixed brain sections were imaged on Zeiss LSM 800 confocal microscope with a $10 \mathrm{X}$ objective (NA 0.45 ).
Image analysis. The time-lapse images were collapsed to make 2D projections using Fluoview 2.1 software. The images of fixed neurons were collapsed to make 2D projections using Zeiss Zen 3.1 software. MetaMorph 6.1 software (Molecular Devices) was used to measure internalized GluA2, surface-remaining GluA2, transferrin, mRFP-LC3, EGFP-Rab5, EGFP-Rab11, LC3 puncta and EGFP-Rab9 on dendrites, and Atg5 and HA in the soma. Briefly, dendrites and soma were traced manually using the Create Region tool. The Threshold Image function was applied to the traced dendrites or soma with the same threshold for all images from one experiment. The Region Measurement tool was used to automatically outline all puncta in the selected area and measure the intensity and size of each puncta. Because previous electron microscopy studies show that the diameter of autophagosomes varies between $300-400 \mathrm{~nm}$ and several micrometers ${ }^{62}$, we excluded LC3 positive puncta with diameters $<300 \mathrm{~nm}$ in the LC3 analysis. To analyze Atg5 immunofluorescence in fixed brain slices, $z$-stack images were collapsed using Zeiss Zen software, then analyzed with ImageJ software. Somas in the pyramidal cell layer of the hippocampal CA1 and CA3 regions were traced manually using the Polygon Area Selection tool in ImageJ followed by image thresholding, with the same threshold for all images in one experimental group consisting of $\mathrm{Cre}^{-}, \mathrm{CA}_{1 C r e}{ }^{+}$and $\mathrm{CA} 3 \mathrm{Cre}^{+}$brains. Integrated Atg5 immunofluorescence intensity in the pyramidal cell layer was measured using the Particle Analysis function in Image J win-64 software and divided by the number of somas to obtain the mean Atg5 immunofluorescence intensity per cell. 

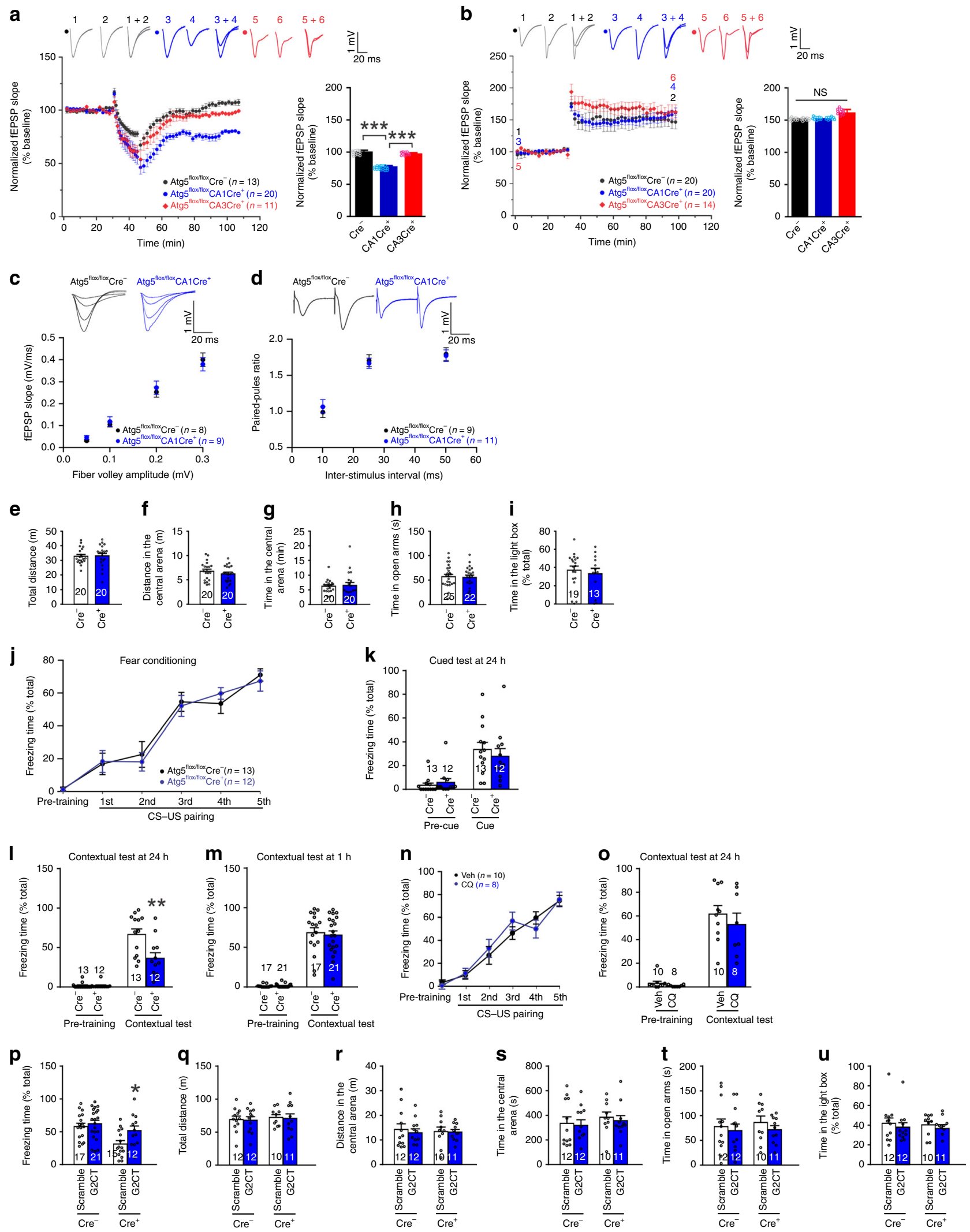

Behavior. 2-4 mice were housed in each cage under a $12 \mathrm{~h}$ light/dark cycle at $21-23.3^{\circ} \mathrm{C}$ and a relative humidity of $35-60 \%$ with food and water ad libitum. 9-12-week-old male littermates were tested for behavior during the dark period. Behavioral data were analyzed using TopScan 3.0 software (CleverSystems). Animal assignment to individual experimental conditions was random.

Open field test. Mice were placed in the center of the test box $(49 \mathrm{~cm} \times 49 \mathrm{~cm} \times$ $40 \mathrm{~cm}$ ) and allowed to freely explore the arena for 30 min The distance traveled in the whole area and the central area $(20 \mathrm{~cm} \times 20 \mathrm{~cm})$ of the box, and the time spent in the central area was analyzed.

Light/dark box test. Mice were placed in the center of the light compartment of a light/dark box $(46 \mathrm{~cm} \times 27 \mathrm{~cm} \times 30 \mathrm{~cm}$, two-thirds were illuminated and one third 
Fig. 10 Contextual fear memory is impaired in CA1-specific Atg 5 knockout mice. LTD induced by LFS (a) and LTP induced by HFS (b) in the hippocampal CA1 region of 8-week-old mice; quantification on the right shows fEPSPs at 50-60 min after LFS or HFS normalized to the pre-stimulation baseline; oneway ANOVA was used for comparison across groups $\left(p=3.8 \times 10^{-28}\right)$ and Student-Newman-Keuls test for comparison with the Cre ${ }^{-}$group. $\mathbf{c}$ The inputoutput relationship. $\mathbf{d}$ Paired-pulse ratio. e-g Open field test. $\mathbf{h}$ Elevated zero maze test. $\mathbf{i}$ Light/dark box test. $\mathbf{j}$ The percentage of time spent in freezing before and after each CS-US pairing. $\mathbf{k}$ Cued fear memory tested at $24 \mathrm{~h}$ after fear conditioning. I Contextual fear memory tested at $24 \mathrm{~h}$ after fear conditioning; two-way ANOVA was used for statistical analysis ( $p=0.005$ for interaction between the effects of genotype and test on freezing; $p=0.004$ for the simple main effect of $\mathrm{Cre}^{-}$vs. $\mathrm{Cre}^{+}$in the contextual test). $\mathbf{m}$ Contextual fear memory tested at $1 \mathrm{~h}$ after fear conditioning. $\mathbf{n}, \mathbf{o}$ Fear conditioning training and contextual memory test at 2 weeks after cannula implantation in the dorsal hippocampal CA1 region of WT mice. CQ was infused through the implanted cannula at 30 min before the contextual fear memory test. p-u Mice were injected with AAV expressing G2CT or scrambled peptides and tested at 3 weeks after injection for contextual fear memory ( $\mathbf{p}$; two-way ANOVA was used for statistical analysis; $p=0.158$ for interaction between the effects of genotype and virus on freezing; $p=0.032$ for the main effect of virus; $p=0.021$ for the comparison between scramble vs. G2CT within Cre ${ }^{+}$using Student-Newman-Keuls test), open field test ( $\mathbf{q}-\mathbf{s})$, elevated zero maze test (t) or light/dark box test (u). Data show mean $\pm \mathrm{SEM}$; ${ }^{\star} p<0.05$, ${ }^{\star \star} p<0.01$, ${ }^{* \star} p<0.001$; no adjustments were made for multiple comparisons; $n$ indicates the number of slices in $\mathbf{a}-\mathbf{d}$ (one slice from each animal) and the number of animals in $\mathbf{e}-\mathbf{u}$. Source data are provided as a Source Data file.

was darkened) and allowed to freely explore the test box for $11 \mathrm{~min}$ The time spent in the light and the dark compartment was analyzed.

Elevated O-maze test. The elevated zero maze $(60 \mathrm{~cm}$ in diameter, Clever Sys Inc.) is divided into four equal sections (two open and two enclosed). Mice were placed in the middle of one open section to begin the test and left on the maze for $6 \mathrm{~min}$ The time spent in the closed and the open sections were analyzed.

Fear conditioning. On day 1 of the experiment, the mouse was habituated to the test chamber (Med Associates) for 2 min On day 2, the mouse was exposed to 5 conditioned stimuli (CS; $6000 \mathrm{~Hz}, 85 \mathrm{~dB}$ tone lasting $30 \mathrm{~s}$, with a random interstimulus interval of 50-150 s), each co-terminated with an unconditioned stimulus (US; electrical foot-shock, $0.5 \mathrm{~s}, 0.75 \mathrm{~mA}$ ). Contextual fear memory was tested at 1 and $24 \mathrm{~h}$ after fear conditioning by placing the mouse in the fear conditioning context for $6 \mathrm{~min}$ Cued fear memory was tested at $24 \mathrm{~h}$ after fear conditioning by exposing the mouse to a tone $(6000 \mathrm{~Hz}, 85 \mathrm{~dB}$, lasting $2 \mathrm{~min})$ in a context different from the fear conditioning context. Freezing was identified by complete immobility (except for respiratory movement) with the Video Freeze 2.15 software (Med Associates).

Statistical analysis. The SigmaPlot 13.0 and IBM SPSS Statistics 26 software was used for statistical analysis. For the comparison of two groups, data were analyzed by two-tailed Student's $t$ test or paired Student's $t$ test. To test for differences among more than two groups, normally distributed data with equal variance were analyzed using one-way ANOVA, one-way RM ANOVA or two-way ANOVA. The data that did not pass the normality and equal variance tests were analyzed using Kruskal-Wallis oneway analysis of variance on ranks. Student-Newman-Keuls test, Bonferroni test, Holm-Sidak test or Dunn's test were used for post hoc analysis to identify significantly different groups. All statistical analysis was two-tailed and $p<0.05$ was considered significant. The specific statistical analysis method for each experiment is described in Figure legends. Sample size was estimated from similar experiments previously done in the lab. No data were excluded from the analysis. All results were produced from $\geq 2$ independent experiments and replicated. All animal assignment and data analyses were done blindly to the experimental condition.

Reporting summary. Further information on research design is available in the Nature Research Reporting Summary linked to this article.

\section{Data availability}

The data that support the findings of this study are provided as a Source Data file. Uncropped immunoblots were provided in Supplementary Fig. 9. Source data are provided with this paper.

\section{Code availability}

No code is generated by this study.

Received: 3 November 2017; Accepted: 18 May 2020;

Published online: 12 June 2020

\section{References}

1. Dong, Z. et al. Mechanisms of hippocampal long-term depression are required for memory enhancement by novelty exploration. J. Neurosci. Off. J. Soc. Neurosci. 32, 11980-11990 (2012).

2. Nicholls, R. E. et al. Transgenic mice lacking NMDAR-dependent LTD exhibit deficits in behavioral flexibility. Neuron 58, 104-117 (2008).

3. Etkin, A. et al. A role in learning for SRF: deletion in the adult forebrain disrupts LTD and the formation of an immediate memory of a novel context. Neuron 50, 127-143 (2006)
4. Griffiths, S. et al. Expression of long-term depression underlies visual recognition memory. Neuron 58, 186-194 (2008).

5. Liu, X., Gu, Q. H., Duan, K. \& Li, Z. NMDA receptor-dependent LTD is required for consolidation but not acquisition of fear memory. J. Neurosci. Off. J. Soc. Neurosci. 34, 8741-8748 (2014).

6. Ge, Y. et al. Hippocampal long-term depression is required for the consolidation of spatial memory. Proc. Natl Acad. Sci. USA 107, 16697-16702 (2010).

7. Dong, Z. et al. Hippocampal long-term depression mediates spatial reversal learning in the Morris water maze. Neuropharmacology 64, 65-73 (2013).

8. Kim, J. I. et al. PI3Kgamma is required for NMDA receptor-dependent longterm depression and behavioral flexibility. Nat. Neurosci. 14, 1447-1454 (2011)

9. Li, J. et al. Synaptic P-Rex1 signaling regulates hippocampal long-term depression and autism-like social behavior. Proc. Natl Acad. Sci. USA 112, E6964-E6972 (2015).

10. Errington, M. L. et al. Stimulation at $1-5 \mathrm{~Hz}$ does not produce long-term depression or depotentiation in the hippocampus of the adult rat in vivo. $J$. Neurophysiol. 74, 1793-1799 (1995).

11. Manahan-Vaughan, D. \& Braunewell, K. H. Novelty acquisition is associated with induction of hippocampal long-term depression. Proc. Natl Acad. Sci. USA 96, 8739-8744 (1999).

12. Norris, C. M., Korol, D. L. \& Foster, T. C. Increased susceptibility to induction of long-term depression and long-term potentiation reversal during aging. $J$. Neurosci.: Off. J. Soc. Neurosci. 16, 5382-5392 (1996).

13. Wagner, J. J. \& Alger, B. E. GABAergic and developmental influences on homosynaptic LTD and depotentiation in rat hippocampus. J. Neurosci. Off. J. Soc. Neurosci. 15, 1577-1586 (1995).

14. Huganir, R. L. \& Nicoll, R. A. AMPARs and synaptic plasticity: the last 25 years. Neuron 80, 704-717 (2013).

15. Jiao, S. \& Li, Z. Nonapoptotic function of BAD and BAX in long-term depression of synaptic transmission. Neuron 70, 758-772 (2011).

16. $\mathrm{Li}, \mathrm{Z}$. et al. Caspase- 3 activation via mitochondria is required for long-term depression and AMPA receptor internalization. Cell 141, 859-871 (2010).

17. Malinow, R. \& Malenka, R. C. AMPA receptor trafficking and synaptic plasticity. Annu Rev. Neurosci. 25, 103-126 (2002).

18. Ehlers, M. D. Reinsertion or degradation of AMPA receptors determined by activity-dependent endocytic sorting. Neuron 28, 511-525 (2000).

19. Kim, J., Kundu, M., Viollet, B. \& Guan, K. L. AMPK and mTOR regulate autophagy through direct phosphorylation of Ulk1. Nat. Cell Biol. 13, 132-141 (2011).

20. Marino, G., Madeo, F. \& Kroemer, G. Autophagy for tissue homeostasis and neuroprotection. Curr. Opin. Cell Biol. 23, 198-206 (2011).

21. Mizushima, N. \& Komatsu, M. Autophagy: renovation of cells and tissues. Cell 147, 728-741 (2011).

22. Moreau, K., Luo, S. \& Rubinsztein, D. C. Cytoprotective roles for autophagy. Curr. Opin. Cell Biol. 22, 206-211 (2010).

23. Galluzzi, L., Pietrocola, F., Levine, B. \& Kroemer, G. Metabolic control of autophagy. Cell 159, 1263-1276 (2014).

24. Stavoe, A. K., Hill, S. E., Hall, D. H. \& Colon-Ramos, D. A. KIF1A/UNC-104 transports ATG-9 to regulate neurodevelopment and autophagy at synapses. Developmental cell 38, 171-185 (2016).

25. Shen, W. \& Ganetzky, B. Autophagy promotes synapse development in Drosophila. J. cell Biol. 187, 71-79 (2009).

26. Tang, G. et al. Loss of mTOR-dependent macroautophagy causes autistic-like synaptic pruning deficits. Neuron 83, 1131-1143 (2014).

27. Hernandez, D. et al. Regulation of presynaptic neurotransmission by macroautophagy. Neuron 74, 277-284 (2012).

28. Yamamoto, A. \& Yue, Z. Autophagy and its normal and pathogenic states in the brain. Annu Rev. Neurosci. 37, 55-78 (2014). 
29. Shehata, M., Matsumura, H., Okubo-Suzuki, R., Ohkawa, N. \& Inokuchi, K. Neuronal stimulation induces autophagy in hippocampal neurons that is involved in AMPA receptor degradation after chemical long-term depression. J. Neurosci.: Off. J. Soc. Neurosci. 32, 10413-10422 (2012).

30. Kimura, T., Suzuki, M. \& Akagi, T. Age-dependent changes in synaptic plasticity enhance tau oligomerization in the mouse hippocampus. Acta Neuropathol. Commun. 5, 67 (2017).

31. Mizushima, N., Yoshimori, T. \& Ohsumi, Y. The role of Atg proteins in autophagosome formation. Annu Rev. Cell Dev. Biol. 27, 107-132 (2011).

32. Kabeya, Y. et al. LC3, GABARAP and GATE16 localize to autophagosomal membrane depending on form-II formation. J. Cell Sci. 117, 2805-2812 (2004).

33. Ichimura, Y. et al. A ubiquitin-like system mediates protein lipidation. Nature 408, 488-492 (2000).

34. Bjorkoy, G. et al. p62/SQSTM1 forms protein aggregates degraded by autophagy and has a protective effect on huntingtin-induced cell death. J. cell Biol. 171, 603-614 (2005).

35. Shibutani, S. T. \& Yoshimori, T. A current perspective of autophagosome biogenesis. Cell Res 24, 58-68 (2014)

36. Mizushima, N., Yamamoto, A., Matsui, M., Yoshimori, T. \& Ohsumi, Y. In vivo analysis of autophagy in response to nutrient starvation using transgenic mice expressing a fluorescent autophagosome marker. Mol. Biol. Cell 15, 1101-1111 (2004).

37. Mizushima, N., Yoshimori, T. \& Levine, B. Methods in mammalian autophagy research. Cell 140, 313-326 (2010).

38. Kabeya, Y. et al. LC3, a mammalian homologue of yeast Apg8p, is localized in autophagosome membranes after processing. EMBO J. 19, 5720-5728 (2000).

39. Ganley, I. G. et al. ULK1.ATG13.FIP200 complex mediates mTOR signaling and is essential for autophagy. J. Biol. Chem. 284, 12297-12305 (2009).

40. Jung, C. H. et al. ULK-Atg13-FIP200 complexes mediate mTOR signaling to the autophagy machinery. Mol. Biol. Cell 20, 1992-2003 (2009).

41. Nakazawa, K. et al. Requirement for hippocampal CA3 NMDA receptors in associative memory recall. Science 297, 211-218 (2002).

42. Tsien, J. Z. et al. Subregion- and cell type-restricted gene knockout in mouse brain. Cell 87, 1317-1326 (1996).

43. Castillo, K. et al. Trehalose delays the progression of amyotrophic lateral sclerosis by enhancing autophagy in motoneurons. Autophagy 9, 1308-1320 (2013).

44. Rodriguez-Navarro, J. A. et al. Trehalose ameliorates dopaminergic and tau pathology in parkin deleted/tau overexpressing mice through autophagy activation. Neurobiol. Dis. 39, 423-438 (2010).

45. Sarkar, S., Davies, J. E., Huang, Z., Tunnacliffe, A. \& Rubinsztein, D. C Trehalose, a novel mTOR-independent autophagy enhancer, accelerates the clearance of mutant huntingtin and alpha-synuclein. J. Biol. Chem. 282, 5641-5652 (2007).

46. Lee, H. J., Yoon, Y. S. \& Lee, S. J. Mechanism of neuroprotection by trehalose: controversy surrounding autophagy induction. Cell Death Dis. 9, 712 (2018).

47. Bredt, D. S. \& Nicoll, R. A. AMPA receptor trafficking at excitatory synapses. Neuron 40, 361-379 (2003).

48. Collingridge, G. L., Isaac, J. T. \& Wang, Y. T. Receptor trafficking and synaptic plasticity. Nat. Rev. Neurosci. 5, 952-962 (2004).

49. Beattie, E. C. et al. Regulation of AMPA receptor endocytosis by a signaling mechanism shared with LTD. Nat. Neurosci. 3, 1291-1300 (2000).

50. Makino, H. \& Malinow, R. AMPA receptor incorporation into synapses during LTP: the role of lateral movement and exocytosis. Neuron 64, 381-390 (2009).

51. Ashby, M. C. et al. Removal of AMPA receptors (AMPARs) from synapses is preceded by transient endocytosis of extrasynaptic AMPARs. J. Neurosci. 24, 5172-5176 (2004).

52. Ravikumar, B., Moreau, K., Jahreiss, L., Puri, C. \& Rubinsztein, D. C. Plasma membrane contributes to the formation of pre-autophagosomal structures. Nat. Cell Biol. 12, 747-757 (2010).

53. Puri, C., Renna, M., Bento, C. F., Moreau, K. \& Rubinsztein, D. C. Diverse autophagosome membrane sources coalesce in recycling endosomes. Cell 154, 1285-1299 (2013).

54. Norman, J. M., Cohen, G. M. \& Bampton, E. T. The in vitro cleavage of the hAtg proteins by cell death proteases. Autophagy 6, 1042-1056 (2010).

55. Kim, J. J. \& Jung, M. W. Neural circuits and mechanisms involved in Pavlovian fear conditioning: a critical review. Neurosci. Biobehav. Rev. 30, 188-202 (2006).

56. Lee, S. H., Liu, L., Wang, Y. T. \& Sheng, M. Clathrin adaptor AP2 and NSF interact with overlapping sites of GluR2 and play distinct roles in AMPA receptor trafficking and hippocampal LTD. Neuron 36, 661-674 (2002).

57. Crozier, R. A., Wang, Y., Liu, C. H. \& Bear, M. F. Deprivation-induced synaptic depression by distinct mechanisms in different layers of mouse visual cortex. Proc. Natl Acad. Sci. USA 104, 1383-1388 (2007).
58. Tang, S. J. et al. A rapamycin-sensitive signaling pathway contributes to longterm synaptic plasticity in the hippocampus. Proc. Natl Acad. Sci. USA 99, 467-472 (2002)

59. Cammalleri, M. et al. Time-restricted role for dendritic activation of the mTOR-p70S6K pathway in the induction of late-phase long-term potentiation in the CA1. Proc. Natl Acad. Sci. USA 100, 14368-14373 (2003).

60. Hou, L. \& Klann, E. Activation of the phosphoinositide 3-kinase-Aktmammalian target of rapamycin signaling pathway is required for metabotropic glutamate receptor-dependent long-term depression. $J$. Neurosci.: Off. J. Soc. Neurosci. 24, 6352-6361 (2004).

61. Mameli, M., Balland, B., Lujan, R. \& Luscher, C. Rapid synthesis and synaptic insertion of GluR2 for mGluR-LTD in the ventral tegmental area. Science 317, 530-533 (2007).

62. Yla-Anttila, P., Vihinen, H., Jokitalo, E. \& Eskelinen, E. L. Monitoring autophagy by electron microscopy in Mammalian cells. Methods Enzymol. 452, 143-164 (2009).

\section{Acknowledgements}

Jiangsu Provincial Department of Education provided H. S's first-year salary (equivalent to $16.5 \%$ of H.S's total fellowship while she worked on this study as a postdoctoral fellow in Section of Synapse Development and Plasticity, National Institute of Mental Health, National Institutes of Health) through the Jiangsu Overseas Research \& Training Program for University Prominent Young \& Middle-aged Teachers. The rest of the funding for this work was provided by the Intramural Research Program of National Institute of Mental Heal, National Institutes of Health (1ZIAMH002881 to Z.L.).

\section{Author contributions}

Z.L. and H.S. designed the experiment and wrote the manuscript that was submitted by H.S. and Z.L. on November 3, 2017. H.S. performed the experiment and data analysis between 2013. 01-2017.12 that are presented in Fig. 1a, b, h (sham, LFS, HFS), i (sham, LFS, HFS); Fig. 2a-d; Fig. 3a (CQ, rapamycin), b (vehicle, CQ, rapamycin); Fig. 4a (sham, NMDA, NMDA+rapamycin, rapamycin), b, c (sham, NMDA, NMDA+rapamycin, rapamycin), d; Fig. 5a-d, h (pre-CQ, post-CQ), i (CQ), j, k; Fig. 8a-f, j, k, q, r; Fig. 9b, d,

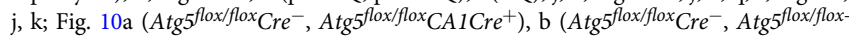
$\left.\mathrm{CA}_{\mathrm{CCr}}{ }^{+}\right), \mathrm{c}, \mathrm{d}, \mathrm{e}-\mathrm{m}, \mathrm{p}$; Supplementary Fig. 1 (D, E, I, J), 2 (D-I), 3A (CQ), 3B (CQ), 4 (A-D), 5, 8 (A-C). H.Z., D.P., Q.G., and Z.L. designed and performed the rest of the experiments, analyzed the resulting data, and wrote the revised manuscript after receiving the reviewers' comments on January 18, 2018.

\section{Competing interests}

The authors declare no competing interests.

\section{Additional information}

Supplementary information is available for this paper at https://doi.org/10.1038/s41467 020-16794-5.

Correspondence and requests for materials should be addressed to Z.L.

Peer review information Nature Communications thanks the anonymous reviewers for their contributions to the peer review of this work.

Reprints and permission information is available at http://www.nature.com/reprints

Publisher's note Springer Nature remains neutral with regard to jurisdictional claims in published maps and institutional affiliations.

Open Access This article is licensed under a Creative Commons Attribution 4.0 International License, which permits use, sharing, adaptation, distribution and reproduction in any medium or format, as long as you give appropriate credit to the original author(s) and the source, provide a link to the Creative Commons license, and indicate if changes were made. The images or other third party material in this article are included in the article's Creative Commons license, unless indicated otherwise in a credit line to the material. If material is not included in the article's Creative Commons license and your intended use is not permitted by statutory regulation or exceeds the permitted use, you will need to obtain permission directly from the copyright holder. To view a copy of this license, visit http://creativecommons.org/ licenses/by/4.0\%

This is a U.S. government work and not under copyright protection in the U.S.; foreign copyright protection may apply 2020 